\title{
Dynamics Modeling of Gear Transmissions with Asymmetric Load-Dependent Friction
}

This paper was downloaded from TechRxiv (https://www.techrxiv.org).

\section{LICENSE}

CC BY 4.0

SUBMISSION DATE / POSTED DATE

$18-01-2022$ / 24-01-2022

CITATION

Kikuuwe, Ryo (2022): Dynamics Modeling of Gear Transmissions with Asymmetric Load-Dependent Friction. TechRxiv. Preprint. https://doi.org/10.36227/techrxiv.18583775.v1

$\mathrm{DOI}$

10.36227/techrxiv.18583775.v1 


\title{
Dynamics Modeling of Gear Transmissions with Asymmetric Load-Dependent Friction
}

\author{
Ryo Kikuuwe, Member, IEEE
}

\begin{abstract}
This paper proposes a new concise mathematical model of gear transmission dynamics with asymmetric loaddependent friction. It is of the form of a differential-algebraic inclusion (DAI) characterized by some parameters, including input-side and output-side asymmetry coefficients. The presented model properly captures the static friction and even the nonbackdrivability. It is applicable to different classes of transmissions, such as leadscrew transmissions, worm gear transmissions, and spur gear transmissions. The DAI representation is extended into a multi-dimensional representation for articulated rigid-body systems driven through joint transmissions. Moreover, simulation algorithms are derived through the implicit Euler discretization. Some simulation examples illustrate the validity of the presented representations.
\end{abstract}

Index Terms - Nonsmooth mechanics, Gear, Friction, Backdrivability

\section{INTRODUCTION}

Gear transmissions are extensively used in robotic mechanisms to extract low speeds and large forces from commercial servo actuators. The modeling of friction in gear transmissions is an important issue for the control and simulation of various robotic systems. Controller design considering the effects of friction is necessary for realizing highly efficient and dynamic motion [1], [2]. Appropriate friction models are also beneficial to force control [3], of which the stability is enhanced by friction compensation [4], [5].

An important feature of the internal friction of gear transmissions is the asymmetry [6], [7]. Specifically, the effect of internal friction is usually smaller when the torque is applied to the input shaft than when it is applied to the output shaft. In an extreme case where the torque applied to the output shaft is blocked by the internal friction, the transmission is said to be non-backdrivable or self-locking [8]. Most high-ratio gear transmissions do not have high backdrivability, which means that there is a large frictional loss when it is driven from the output shaft. The asymmetric frictional loss is also understood in terms of the load dependency [9] because the effect of friction depends on the balance between the torques on the input and output shafts.

Previous modeling approaches of the internal friction considering the asymmetry are based on the Coulomb friction model, with which the friction force between components is proportional to the normal force on the contact surface. Researchers investigated the friction of leadscrew transmissions [10], worm gear transmissions [9]-[12], and spur gear

Machinery Dynamics Laboratory, Hiroshima University, 1-4-1 Kagamiyama, Higashi-Hiroshima, Hiroshima 739-8527, Japan. transmissions [6], [7], [13]. In many of these previous approaches, different expressions must be switched between the forward-driving and backdriving cases, which are defined by the direction of the power flow. Some researchers [2], [6], [10], [11], [14] use different inertia values between the forwarddriving and backdriving cases. Most previous methods cannot handle the static friction state, where the direction of the power flow cannot be defined.

This paper proposes a new mathematical representation of the dynamics of gear transmissions with asymmetric frictional loss. The proposed representation is of the form of a differential-algebraic inclusion (DAI $)^{1}$ with a Lagrange multiplier representing the internal force. It is characterized by some parameters, such as input-side and output-side asymmetry coefficients. The DAI representation is then extended into a multi-dimensional representation for articulated rigidbody systems driven through joint transmissions. This paper also derives simulation algorithms based on the DAI through the implicit Euler discretization. Some numerical examples illustrate its application to robotic simulation.

The remainder of this paper is organized as follows. Section II provides some mathematical preliminaries. Section III presents the main result of the work, which is the new representation of the dynamics of frictional gear transmissions. Section IV investigates the dynamic and static properties of the presented representations. Section V shows the extension of the model to robotic systems having multiple degrees of freedom. Section VI shows some numerical examples. Section VII provides concluding remarks.

\section{MAThematical PREliminaries}

Throughout this paper, $\mathbb{R}$ stands for the set of all real numbers, $\mathcal{B} \triangleq[-1,1] \subset \mathbb{R}$ is the closed unit ball in $\mathbb{R}, 0$ denotes the zero vector or zero matrix of appropriate dimensions, conv $\mathcal{X}$ stands for the convex hull of $\mathcal{X}$, and $2^{\mathcal{X}}$ is the set of all subsets of $\mathcal{X}$. The notation $f: \mathcal{X}_{0} \rightarrow \mathcal{X}_{1}$ means that $f$ is a single-valued function from the set $\mathcal{X}_{0}$ to the set $\mathcal{X}_{1}$, and $f: \mathcal{X}_{0} \rightrightarrows \mathcal{X}_{1}$ means that $f$ is a set-valued function from the set $\mathcal{X}_{0}$ to the set $\mathcal{X}_{1}$, i.e., $f: \mathcal{X}_{0} \rightrightarrows \mathcal{X}_{1}$ stands for $f: \mathcal{X}_{0} \rightarrow 2^{\mathcal{X}_{1}}$. When a function $f: \mathcal{X}_{0} \rightarrow \mathcal{X}_{1}$ or $f: \mathcal{X}_{0} \rightrightarrows \mathcal{X}_{1}$ is called with a set $\mathcal{X}_{s} \subset \mathcal{X}_{0}$ as its argument, it should be read as follows:

$$
f\left(\mathcal{X}_{s}\right)=\bigcup_{x \in \mathcal{X}_{s}} f(x)=\left\{f(x) \in \mathcal{X}_{1} \mid x \in \mathcal{X}_{s}\right\} \subset \mathcal{X}_{1}
$$

${ }^{1}$ In this paper, a DAI stands for a system of equations that involves at least one differential inclusion (DI) and at least one variable that is algebraically determined. A DI stands for a pair of mathematical expressions connected by " $\in$ " instead of "=." 
This implies that, with a continuous and monotone function $f: \mathbb{R} \rightarrow \mathbb{R}$, one has $f(\mathcal{B})=[f(-1), f(1)]$.

We define the set-valued signum function sgn : $\mathbb{R} \rightrightarrows \mathcal{B}$ and the projection function proj : $2^{\mathbb{R}} \times \mathbb{R} \rightarrow \mathbb{R}$ as follows:

$$
\begin{aligned}
\operatorname{sgn}(x) & \triangleq \begin{cases}x /|x| & \text { if } x \neq 0 \\
\mathcal{B} & \text { if } x=0\end{cases} \\
\operatorname{proj}(\mathcal{X}, x) & \triangleq \underset{\xi \in \mathcal{X}}{\operatorname{argmin}}|\xi-x| .
\end{aligned}
$$

Note that, for $a \leq b$,

$$
\operatorname{proj}([a, b], x)=\max (a, \min (x, b))
$$

holds. With a continuous and monotone function $f: \mathcal{B} \rightarrow \mathbb{R}$, the following statement holds true:

$$
y-x \in-f(\operatorname{sgn}(y)) \Longleftrightarrow y=x-\operatorname{proj}(f(\mathcal{B}), x) .
$$

It can be easily proven as follows:

$$
\begin{aligned}
y-x \in-f(\operatorname{sgn}(y)) \\
\Longleftrightarrow(0<y=x-f(1)) \vee(0>y=x-f(-1)) \\
\quad \vee(0=y \in x-[f(-1), f(1)]) \\
\Longleftrightarrow \quad(y=x-f(1) \wedge x>f(1)) \\
\quad \vee(y=x-f(-1) \wedge x<f(-1)) \\
\quad \vee(y=0 \wedge x \in[f(-1), f(1)]) \\
\Longleftrightarrow y=x-\operatorname{proj}(f(\mathcal{B}), x) .
\end{aligned}
$$

With a vector $\boldsymbol{x} \in \mathbb{R}^{n},\|\boldsymbol{x}\|$ stands for the 2-norm of $\boldsymbol{x}$ (i.e., $\|\boldsymbol{x}\|=\sqrt{\boldsymbol{x}^{T} \boldsymbol{x}} \in \mathbb{R}$ ) and $|\boldsymbol{x}|$ stands for the element-wise absolute-value operator (i.e., $|\boldsymbol{x}|=\left[\left|x_{1}\right|, \cdots,\left|x_{n}\right|\right]^{T} \in \mathbb{R}^{n}$ ). We also use the element-wise signum function sgn : $\mathbb{R}^{n} \rightrightarrows$ $\mathcal{B}^{n}$ defined as follows:

$$
\begin{gathered}
\operatorname{sgn}(\boldsymbol{x}) \triangleq\left\{\left[s_{1}, \cdots, s_{n}\right]^{T} \in \mathcal{B}^{n} \mid s_{1} \in \operatorname{sgn}\left(x_{1}\right) \wedge \cdots\right. \\
\left.\wedge s_{n} \in \operatorname{sgn}\left(x_{n}\right)\right\} .
\end{gathered}
$$

Note that $\mathcal{B}^{n}$ is an $n$-dimensional hypercube, not a unit ball. For brevity, this paper uses $[\boldsymbol{x}] \triangleq \operatorname{diag}(\boldsymbol{x}) \in \mathbb{R}^{n \times n}$ for $\boldsymbol{x} \in$ $\mathbb{R}^{n}$. Note that the following relation holds:

$$
[|x|] \operatorname{sgn}(x)=[\operatorname{sgn}(x)]|x|=x .
$$

With a matrix $\boldsymbol{X} \in \mathbb{R}^{m \times n}, \sigma_{\max }(\boldsymbol{X})$ stands for the maximum singular value of $\boldsymbol{X}$. The following statement holds:

$$
\sigma_{\max }(\boldsymbol{X})=\max _{\boldsymbol{x} \in \mathbb{R}^{n} \backslash 0}\|\boldsymbol{X} \boldsymbol{x}\| /\|\boldsymbol{x}\| .
$$

It should also be noted that the infinity norm of a vector $x$ satisfies $\|\boldsymbol{x}\|_{\infty}=\sigma_{\max }([\boldsymbol{x}])$.

\section{DAI REPRESENTATION OF A GEAR TRANSMISSION}

\section{A. Main Result}

As a model of a gear transmission having asymmetric loaddependent friction, let us consider the system illustrated in Fig. 1. In this system, two carts, representing the input and output shafts of a transmission, slide against each other. The contact between the carts is assumed to be bilateral, i.e., the normal force can be either positive or negative. The moving directions of the carts are at the angles of $\theta_{u}$ and $\theta_{v}$ from the tangential direction of the contact surface. Similar models

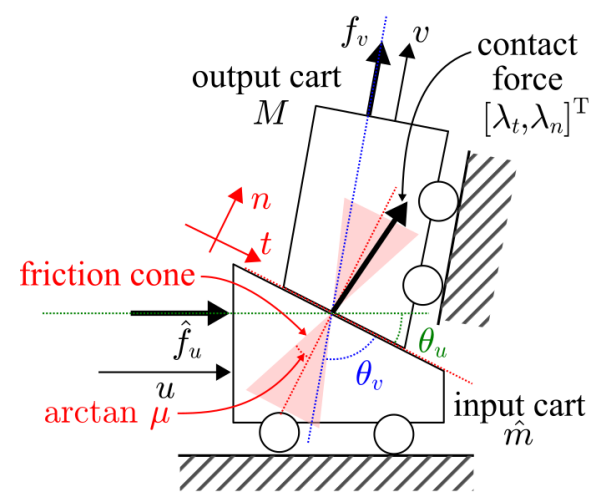

Fig. 1. A model of a gear transmission with asymmetric friction.

have also been investigated in previous work, e.g., [2], [9], [10], [14], [15], often being intended as a simplified model of general gear transmissions. It should be noted that this paper does not consider the backlash in contrast to [14].

As illustrated in Fig. 1, let $\hat{m}$ be the inertia of the input cart, $M$ be the inertia of the output cart, $u \in \mathbb{R}$ be the input velocity, $v \in \mathbb{R}$ be the output velocity, $\hat{f}_{u} \in \mathbb{R}$ be the force acting on the input cart, and $f_{v} \in \mathbb{R}$ be the force from the output cart to the external load. Without loss of generality, we assume that $\theta_{u} \in(0, \pi / 2]$ and $\theta_{v} \in(0, \pi / 2]$. The geometric constraint between the input and output carts can be written as follows:

$$
v=u / \kappa, \dot{v}=\dot{u} / \kappa
$$

where $\kappa \triangleq \sin \theta_{v} / \sin \theta_{u}$. The equations of motion of the carts can be written as follows:

$$
\begin{aligned}
\hat{m} \dot{u} & =\hat{f}_{u}-\lambda_{t} \cos \theta_{u}-\lambda_{n} \sin \theta_{u} \\
M \dot{v} & =-f_{v}-\lambda_{t} \cos \theta_{v}+\lambda_{n} \sin \theta_{v} .
\end{aligned}
$$

Here, $\left[\lambda_{t}, \lambda_{n}\right]^{T}$ is the contact force acting from the input cart to the output cart. The normal component $\lambda_{n}$ is determined to enforce the constraint (10). The tangential component $\lambda_{t}$ is determined by the dry friction at the contact surface as follows:

$$
\lambda_{t} \in \mu\left|\lambda_{n}\right| \operatorname{sgn}(u)
$$

where $\mu$ is the friction coefficient at the contact surface. Throughout this paper, it is assumed that there is no difference between the maximum static friction coefficient and the kinetic friction coefficient. The expression (12) states that the tangential component $\lambda_{t}$ is within the interval $\left[-\mu\left|\lambda_{n}\right|, \mu\left|\lambda_{n}\right|\right]$ in the static friction and is on the boundary of the interval in the kinetic friction.

Solving (11) with respect to $\lambda_{t}$ and $\lambda_{n}$ considering the constraint (10) yields the following expression:

$$
\begin{aligned}
\lambda_{t}= & -\frac{\sin \theta_{u}}{\sin \left(\theta_{u}+\theta_{v}\right)}\left(\left(\kappa^{2} \hat{m}+M\right) \dot{v}-\kappa \hat{f}_{u}-f_{v}\right) \\
\lambda_{n}=- & \frac{\sin \theta_{u}}{\sin \left(\theta_{u}+\theta_{v}\right)} \times \\
& \quad\left(\left(\frac{\kappa^{2} \hat{m}}{\tan \theta_{v}}-\frac{M}{\tan \theta_{u}}\right) \dot{v}-\frac{\kappa \hat{f}_{u}}{\tan \theta_{v}}-\frac{f_{v}}{\tan \theta}\right) .
\end{aligned}
$$




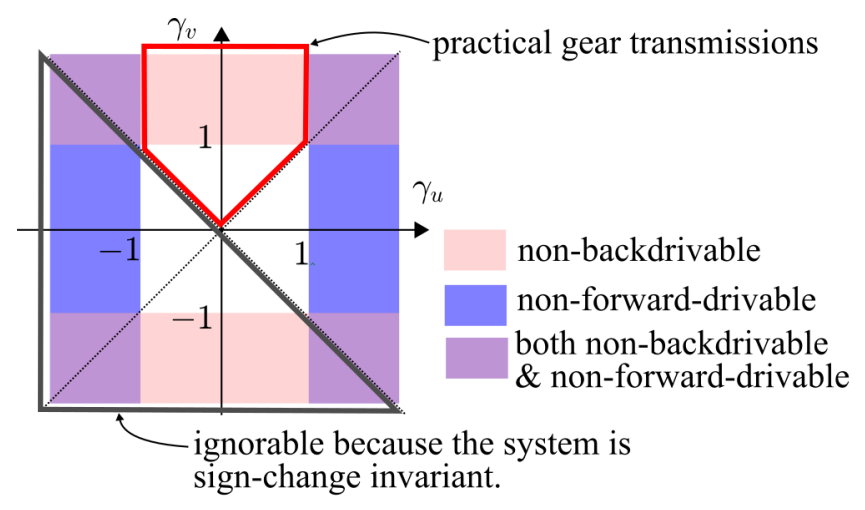

Fig. 2. Backdrivability and forward-drivability in relation to the asymmetry coefficients $\gamma_{u}$ and $\gamma_{v}$.

By setting $m \triangleq \kappa^{2} \hat{m}, f_{u} \triangleq \kappa \hat{f}_{u}, \gamma_{u} \triangleq \mu / \tan \theta_{v}, \gamma_{v} \triangleq$ $\mu / \tan \theta_{u}$, and $\lambda \triangleq \mu \lambda_{n} \sin \left(\theta_{u}+\theta_{v}\right) / \sin \theta_{u}$ and considering (12), one can rewrite (13) as follows:

$$
\begin{aligned}
(m+M) \dot{v} & \in f_{u}-f_{v}-|\lambda| \operatorname{sgn}(v) \\
\left(\gamma_{u} m-\gamma_{v} M\right) \dot{v} & =\gamma_{u} \kappa f_{u}+\gamma_{v} f_{v}-\lambda .
\end{aligned}
$$

Note that (14) is a DAI and it is the equation of motion of the system of Fig. 1.

The DAI representation (14) is the main result of this paper. Although (14) is derived from the specific model in Fig. 1, it is general enough to describe different types of gear transmissions as will be shown in subsequent sections. The system (14) can be seen as a nonsmooth differentialalgebraic system with the state $v$, the inputs $\left\{f_{u}, f_{v}\right\}$, and the Lagrange multiplier $\lambda$, and is characterized by the following four parameters:

- $\gamma_{u} \in \mathbb{R}$ : the input-side "asymmetry coefficient,"

- $\gamma_{v} \in \mathbb{R}:$ the output-side "asymmetry coefficient,"

- $m \in(0, \infty)$ : the input-side inertia, and

- $M \in(0, \infty)$ : the output-side inertia.

One may call (14a) as a visible dynamics that determines the acceleration $\dot{v}$ and (14b) as a hidden dynamics that determines the internal force $\lambda$. When $\gamma_{u}=\gamma_{v}=0$, i.e., when there is no friction between the carts, the hidden dynamics (14b) vanishes and the whole system (14) reduces to an ordinary differential equation $(m+M) \dot{v}=f_{u}-f_{v}$, which is quite a conventional representation.

It should be noted that the system (14) is invariant to the sign changes of the pair $\left\{\gamma_{u}, \gamma_{v}\right\}$ because it only reverses the sign of $\lambda$ and does not affect $\dot{v}$. Thus, without loss of generality, we can assume that $\gamma_{u}+\gamma_{v} \geq 0$.

If $\left|\gamma_{v}\right| \geq 1$, one can say that the transmission is nonbackdrivable (or self-locking) because setting $f_{u}=v=\dot{v}=0$ with (14) results in $\lambda=\gamma_{v} f_{v}$ and $0 \in-f_{v}-\left|\gamma_{v} f_{v}\right| \mathcal{B}$, which holds true with any $f_{v} \in \mathbb{R}$ if $\left|\gamma_{v}\right| \geq 1$. This means that, when $f_{u}=0$, any large force $f_{v}$ applied to the output shaft does not produce any movements at the static friction state if $\left|\gamma_{v}\right| \geq 1$. In the same light, one may say that the transmission is non-forward-drivable if $\left|\gamma_{u}\right| \geq 1$, although it is not the case with most practical gear transmissions. Fig. 2 shows the map of the backdrivability and the forward-drivability in the $\gamma_{u}-\gamma_{v}$

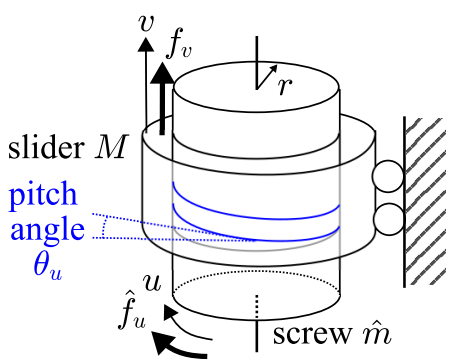

Fig. 3. Leadscrew transmission.

plane. Section IV-A will present a more detailed discussion to relate the coefficients $\gamma_{u}$ and $\gamma_{v}$ to the backward/forward efficiency of the transmission.

By setting $\theta_{u}+\theta_{v}=\pi / 2$, this model reduces to the model discussed in [15, Section 5.5.5] except that the contact between these carts is a bilateral constraint instead of a unilateral constraint. The case of $\theta_{u}+\theta_{v}=\pi / 2$ is discussed also in [9]. In contrast to the proposed representation (14), the expressions presented in [9] involve explicit switching of whether the normal force is positive or negative,

\section{B. Example 1: Leadscrew Transmission}

The proposed DAI representation (14) describes some different types of transmissions. The following sections present some examples.

A leadscrew transmission such as those illustrated in Fig. 3 can be seen as one of the simplest examples. Let us assume that its lead angle is $\theta_{u}$, the screw radius is $r$, and the friction coefficient is $\mu$. Let $\hat{m}$ be the moment of inertia of the input screw and $M$ be the inertia of the output slider. Let $u$ be the angular velocity of the input screw and $v$ be the linear velocity of the output slider. Then, the system of Fig. 3 becomes equivalent to the system of Fig. 1 with $u$ and $\theta_{v}$ being replaced by $r u$ and $\pi / 2-\theta_{u}$, respectively. (See also [10, Fig. 2].) Therefore, one can see that the dynamics of a leadscrew transmission in Fig. 3 can be described by the proposed DAI representation (14) with $m=\kappa^{2} \hat{m}, f_{u}=\kappa \hat{f}_{u}$, $\kappa \triangleq r / \tan \theta, \gamma_{u} \triangleq \mu \tan \theta$, and $\gamma_{v} \triangleq \mu / \tan \theta$.

\section{Example 2: Worm Gear Transmission}

As another example, let us consider a worm gear transmission illustrated in Fig. 4, which has been investigated also in [11], [12]. Partially borrowing Yeh and Wu's [11] notations, let $\ell_{w}>0$ and $\ell_{g}>0$ be the pitch radii of the worm and the gear, respectively, $\phi_{n} \in(0, \pi / 2)$ be the pressure angle, and $\lambda_{l} \in(0, \pi / 4]$ be the lead angle. Other symbols, $u, v, \hat{f}_{u}, f_{v}$, $\hat{m}$, and $M$, are defined in the same manner as in Section III-A and Fig. 1.

Yeh and $\mathrm{Wu}$ [11] have derived two sets of equations of motion of this system; one is for the "left engagement" case and the other is for the "right engagement" case. These cases correspond to the cases where the force from the worm to the gear in Fig. 4 is rightward and leftward, respectively. Borrowing their notations, let $W_{n}>0$ be the magnitude of 


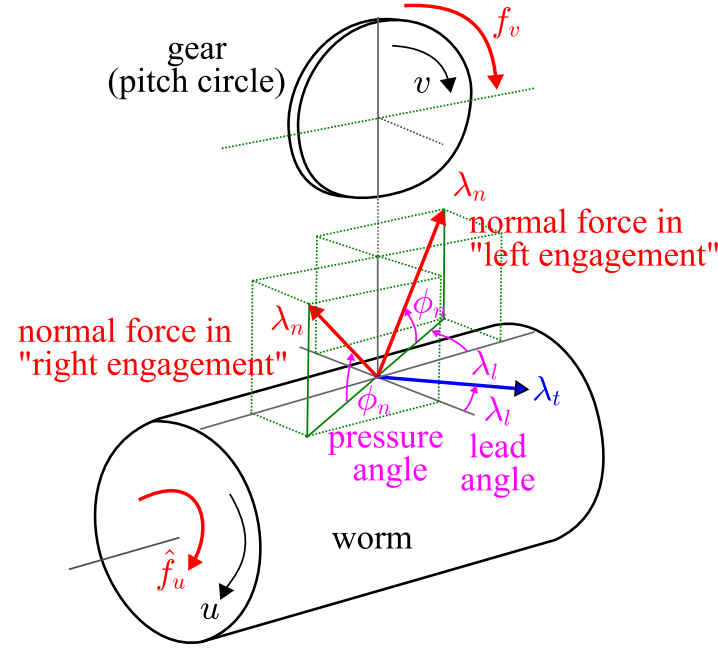

Fig. 4. Worm gear transmission, adapted from [11].

the normal component of the contact force. Then, by setting $\lambda_{n}=W_{n}$ in the "left engagement" and $\lambda_{n}=-W_{n}$ in the "right engagement," Eqs. (2) and (3) in [11] can be unified into the following form:

$$
\begin{aligned}
& \hat{m} \dot{u}=\hat{f}_{u}+\left(\lambda_{n} \cos \phi_{n} \sin \lambda_{l}-W_{f} \cos \lambda_{l}\right) \ell_{w} \\
& M \dot{v}=-f_{v}-\left(\lambda_{n} \cos \phi_{n} \cos \lambda_{l}+W_{f} \sin \lambda_{l}\right) \ell_{g}
\end{aligned}
$$

where $W_{f}$ is the tangential component of the contact force. The Coulomb friction law results in $W_{f} \in \mu\left|\lambda_{n}\right| \operatorname{sgn}(u)$, and the geometric constraint can be written as $v=u / \kappa$ where $\kappa=\ell_{g} /\left(\ell_{w} \tan \lambda_{l}\right)$. Thus, solving (15) results in

$$
\begin{aligned}
\lambda_{t} & =\hat{m} \dot{u}+M \dot{v} \tan \lambda_{l}-\hat{f}_{u} \tan \lambda_{l}-f_{v} \\
\lambda_{n} & =\hat{m} \dot{u} \tan \lambda_{l}-M \dot{v}-\hat{f}_{u} \tan \lambda_{l}-f_{v} .
\end{aligned}
$$

One can see that (16) reduces to the proposed DAI representation (14) by setting

$$
\kappa=\frac{\ell_{g}}{\ell_{w} \tan \lambda_{l}}, \gamma_{u}=-\frac{\mu \tan \lambda_{l}}{\cos \phi_{n}}, \gamma_{v}=\frac{\mu}{\tan \lambda_{l} \cos \phi_{n}} .
$$

The model derived by Yeh and $\mathrm{Wu}$ [11] consists of four different equations of motion that must be appropriately switched according to the engagement (left or right) of the gears and the direction (positive or negative) of the velocity $u$. In contrast, the presented formalism (14) is a single unified expression that always holds, even in the case of static friction.

\section{Example 3: Spur-Gear Transmission}

We can also see that the proposed DAI representation (14) can be applied to describe spur-gear transmissions. Let us consider a spur gear transmission of Fig. 5, where $R_{u}$ and $R_{v}$ are the pitch radii of the input and output gears, respectively, and $\alpha$ is the pressure angle. When the origin is placed at the pitch point and the $x$-axis is aligned to the line connecting the rotation centers of the gears, the coordinates of the input shaft and the output shaft are $\left[-R_{u}, 0\right]^{T}$ and $\left[R_{v}, 0\right]^{T}$, respectively. As a property of the spur gear geometry, the contact points are located on the two lines making the angle $\alpha$ with the $y$

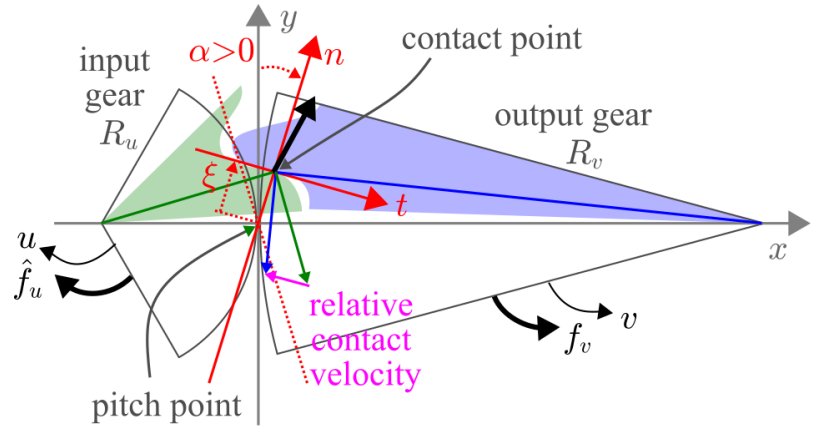

Fig. 5. Spur-gear transmission.

axis at the origin, i.e., their coordinates are $\xi[\sin \alpha, \cos \alpha]^{T}$ or $\xi[-\sin \alpha, \cos \alpha]^{T}$ where $\xi \in \mathbb{R}$. The unit vectors along the tangential and normal directions at a contact point are $[\cos \alpha,-\sin \alpha]^{T}$ and $[\sin \alpha, \cos \alpha]^{T}$, respectively.

Let us consider the case where there is only one contact point $\xi[\sin \alpha, \cos \alpha]^{T}$. Let the contact force from the input gear to the output gear be denoted by $\left[\lambda_{x}, \lambda_{y}\right]^{T}$. Then, the equations of motion of the gears are written as follows:

$$
\begin{aligned}
\hat{m} \dot{u} & =\hat{f}_{u}-\left|\left[\xi \sin \alpha+R_{u}, \xi \cos \alpha\right]^{T},-\left[\lambda_{x}, \lambda_{y}\right]^{T}\right| \\
M \dot{v} & =-f_{v}+\left|\left[\xi \sin \alpha-R_{v}, \xi \cos \alpha\right]^{T},\left[\lambda_{x}, \lambda_{y}\right]^{T}\right|
\end{aligned}
$$

where $\left|[a, b]^{T},[c, d]^{T}\right|=a d-b c$. The positive directions of $u$ and $v$ are clockwise and counter-clockwise, respectively. The tangential and normal components $\left\{\lambda_{t}, \lambda_{n}\right\}$ of the contact force is given as follows:

$$
\left[\begin{array}{c}
\lambda_{t} \\
\lambda_{n}
\end{array}\right]=\left[\begin{array}{cc}
\cos \alpha & -\sin \alpha \\
\sin \alpha & \cos \alpha
\end{array}\right]\left[\begin{array}{l}
\lambda_{x} \\
\lambda_{y}
\end{array}\right] .
$$

Eliminating $\left\{\lambda_{x}, \lambda_{y}\right\}$ from (18) and (19) results in the following:

$$
\begin{aligned}
\lambda_{t} & =-\frac{(m+M) \dot{v}-\kappa f_{u}+f_{v}}{\xi(1+\kappa)} \\
\lambda_{n} & =\frac{\left(\gamma_{u} m-\gamma_{v} M\right) \dot{v}-\gamma_{u} f_{u}-\gamma_{v} f_{v}}{\mu|\xi|(1+\kappa)}
\end{aligned}
$$

where $m \triangleq \kappa^{2} \hat{m}, f_{u} \triangleq \kappa \hat{f}_{u}, \kappa \triangleq R_{v} / R_{u}$, and

$$
\begin{aligned}
& \gamma_{u} \triangleq-\operatorname{sgn}(\xi) \mu \tan \alpha+\frac{\mu|\xi|}{R_{v} \cos \alpha} \\
& \gamma_{v} \triangleq \operatorname{sgn}(\xi) \mu \tan \alpha+\frac{\mu|\xi|}{R_{u} \cos \alpha} .
\end{aligned}
$$

Due to the Coulomb friction law, $\lambda_{t}$ and $\lambda_{n}$ are related by the following:

$$
\lambda_{t} \in-\mu\left|\lambda_{n}\right| \operatorname{sgn}(\xi) \operatorname{sgn}(u) .
$$

Considering (20) and (22), we can obtain the proposed DAI representation (14) with $\lambda=-\mu|\xi|(1+\kappa) \lambda_{n}$ and $\left\{\gamma_{u}, \gamma_{v}\right\}$ defined in (21).

At every given instant, the equation of motion of this system is the DAI (14) but the coefficients $\left\{\gamma_{u}, \gamma_{v}\right\}$ in (21) depend on the location of the contact point, which varies as the gears rotate. The number of contact points also varies between one and two as the gears rotate. By integrating $\left\{\gamma_{u}, \gamma_{v}\right\}$ in 


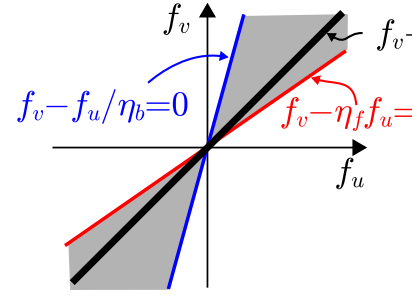

BD \& FD

$\gamma_{u} \in[-1,1], \gamma_{v} \in[-1,1]$

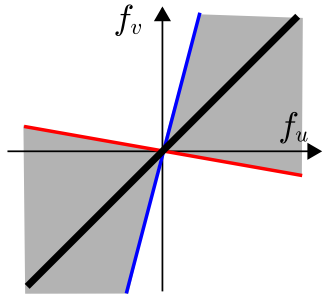

$\mathrm{BD} \& \mathrm{nFD}$
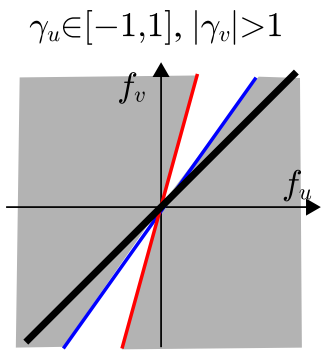

$\mathrm{nBD} \& \mathrm{nFD}$

$\left|\gamma_{u}\right|>\left|\gamma_{v}\right|>1, \gamma_{u} \gamma_{v}<0$

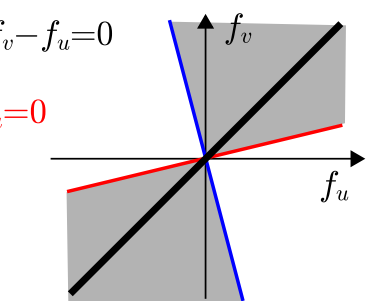

$\mathrm{nBD} \& \mathrm{FD}$
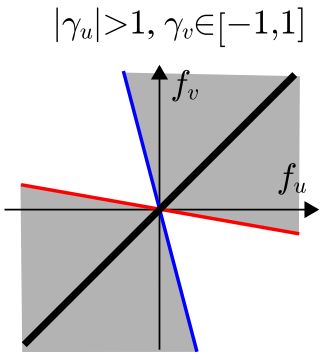

$\mathrm{nBD} \& \mathrm{nFD}$

$\left|\gamma_{u}\right|>1,\left|\gamma_{v}\right|>1, \gamma_{u} \gamma_{v}>0$

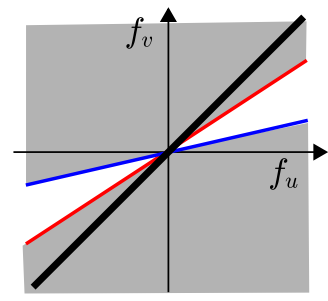

$\mathrm{nBD} \& \mathrm{nFD}$

$\left|\gamma_{v}\right|>\left|\gamma_{u}\right|>1, \gamma_{u} \gamma_{v}<0$
Fig. 6. The set of $\left[f_{u}, f_{v}\right]^{T}$ that satisfy (25), with which the system (14) can be in the static friction state $(\mathrm{BD}=$ backdrivable, $\mathrm{nBD}=$ non-backdrivable, $\mathrm{FD}=$ forward-drivable, $\mathrm{nFD}=$ non-forward-drivable $)$.

(21) with respect to $\xi$ over a full contact cycle, one may obtain average values of the coefficients $\left\{\gamma_{u}, \gamma_{v}\right\}$ with some assumptions on the gear teeth geometry in a similar manner to those in [6], [7], [13], [16]. Another approach to obtain $\left\{\gamma_{u}, \gamma_{v}\right\}$ may be from empirical measurements of the torques in both shafts in the forward-driving and backdriving cases, as discussed in the upcoming Section IV-A.

\section{Properties ANd USE of DAI (14)}

This section discusses some properties of the nonsmooth differential-algebraic system (14).

\section{A. Statics}

Let us consider the case where the system (14) is in the static equilibrium, i.e., $v=0$ and $\dot{v}=0$. This situation can also be said to be the static friction state. In this case, (14) reduces to the following:

$$
f_{u}-f_{v} \in\left|\gamma_{u} f_{u}+\gamma_{v} f_{v}\right| \mathcal{B} .
$$

From some straightforward derivations, (23) can be rewritten as follows:

$$
\begin{aligned}
& \left(\left(1+\gamma_{v}\right) f_{v}-\left(1-\gamma_{u}\right) f_{u}\right) \times \\
& \quad\left(\left(1+\gamma_{u}\right) f_{u}-\left(1-\gamma_{v}\right) f_{v}\right) \geq 0 .
\end{aligned}
$$

Neglecting the singular case of $1+\gamma_{v}=0 \vee 1+\gamma_{u}=0$, one can rewrite (24) as follows:

$$
\left(1+\gamma_{u}\right)\left(1+\gamma_{v}\right)\left(f_{v}-\eta_{f} f_{u}\right)\left(f_{u}-\eta_{b} f_{v}\right) \geq 0
$$

where

$$
\eta_{f} \triangleq \frac{1-\gamma_{u}}{1+\gamma_{v}}, \quad \eta_{b} \triangleq \frac{1-\gamma_{v}}{1+\gamma_{u}} .
$$

Fig. 6 illustrates the set of $\left[f_{u}, f_{v}\right]^{T}$ that satisfy the condition (25) of the static friction. In the case where $\left|\gamma_{u}\right|<1 \wedge\left|\gamma_{v}\right|<1$, i.e., where the transmission is both backdrivable and forwarddrivable, we have $\eta_{f} \in(0,1)$ and $\eta_{b} \in(0,1)$ from (26). In this case, the condition $f_{v}=\eta_{f} f_{u}$, which is one of the critical conditions of (25), takes place in the marginally forward-driving situation where the input shaft is driven with the torque $f_{u}$ and the output shaft applies the torque $f_{v}$ to the external load. Thus, $\eta_{f}$ can be seen as the forward efficiency of the transmission. Conversely, the other critical condition $f_{u}=\eta_{b} f_{v}$ of (25) takes place in the marginally backdriving situation where the transmission is driven from the output shaft. Thus, $\eta_{b}$ can be interpreted as the backward efficiency of the transmission.

The solution of (26) with respect to $\left\{\gamma_{u}, \gamma_{v}\right\}$ is the following:

$$
\gamma_{u}=\frac{1-2 \eta_{f}+\eta_{b} \eta_{f}}{1-\eta_{b} \eta_{f}}, \gamma_{v}=\frac{1-2 \eta_{b}+\eta_{b} \eta_{f}}{1-\eta_{b} \eta_{f}} .
$$

In practice, the values of the forward and backward efficiencies $\left\{\eta_{f}, \eta_{b}\right\}$ can be obtained by some experiments or are provided by the manufacturers. The asymmetry coefficients $\left\{\gamma_{u}, \gamma_{v}\right\}$ to be used in the DAI representation (14) can be obtained from the efficiencies $\left\{\eta_{f}, \eta_{b}\right\}$ through (27).

In case where the transmission is non-backdrivable or nonforward-drivable, i.e., where $\left|\gamma_{u}\right|>1 \vee\left|\gamma_{v}\right|>1$, the quantities $\eta_{f}$ and $\eta_{b}$ can have values outside the interval $(0,1)$. In such a case, we do not have convenient interpretations of these coefficients, except that they are the slopes of the lines in Fig. 6. We can see from Fig. 6 that being both nonforward-drivable and non-backdrivable does not mean that the transmission is non-drivable, but means that it can be driven only if the input and output torques are applied in appropriate ratios.

\section{B. Dynamics}

The dynamics of the nonsmooth differential-algebraic system (14) is now investigated. We can see that (14) can be equivalently rewritten as follows:

$$
\begin{aligned}
(m+M) \dot{v} & =f_{u}-f_{v}-s|\lambda| \\
\lambda-\hat{\rho} s|\lambda| & =\gamma_{u} f_{u}+\gamma_{v} f_{v}-\hat{\rho}\left(f_{u}-f_{v}\right) \\
s & \in \operatorname{sgn}(v)
\end{aligned}
$$

where

$$
\hat{\rho} \triangleq \frac{\gamma_{u} m-\gamma_{v} M}{m+M} .
$$

Lemma 1 in Appendix A suggests that (28) is equivalently rewritten as follows:

$$
(m+M) \dot{v}=f_{u}-f_{v}-s|\lambda|
$$




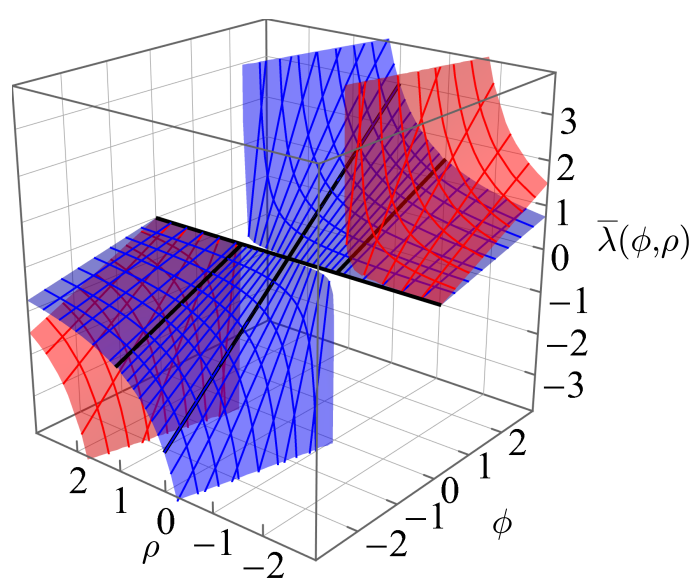

Fig. 7. Set-valued function $\bar{\lambda}$ defined in (31), both blue and red surfaces. If $\phi=0 \vee|\rho|<1, \bar{\lambda}(\phi, \rho)$ is single-valued. If $\phi \neq 0 \wedge|\rho|>1, \bar{\lambda}(\phi, \rho)$ is either empty-valued or dual-valued. The single-valued function $\bar{\lambda}_{s}$ defined in (35) consists only of the blue surface in this figure.

$$
\begin{aligned}
& \lambda \in \bar{\lambda}\left(\bar{\phi}\left(f_{u}, f_{v}, \hat{\rho}\right), \hat{\rho} s\right) \\
& s \in \operatorname{sgn}(v)
\end{aligned}
$$

where $\bar{\lambda}: \mathbb{R} \times \mathbb{R} \rightrightarrows \mathbb{R}$ and $\bar{\phi}: \mathbb{R} \times \mathbb{R} \times \mathbb{R} \rightarrow \mathbb{R}$ are defined as follows:

$$
\begin{aligned}
& \bar{\lambda}(\phi, \rho) \triangleq \begin{cases}0 & \text { if } \phi=0 \\
\phi /(1-\operatorname{sgn}(\phi) \rho) & \text { if } \phi \neq 0 \wedge|\rho|<1 \\
\left\{\frac{\phi}{1-\rho}, \frac{\phi}{1+\rho}\right\} & \text { if } \phi \rho<0 \wedge|\rho|>1 \\
\phi / 2 & \text { if } \phi \rho<0 \wedge|\rho|=1 \\
\emptyset & \text { if } \phi \rho>0 \wedge|\rho| \geq 1\end{cases} \\
& \bar{\phi}\left(f_{u}, f_{v}, \rho\right) \triangleq \gamma_{u} f_{u}+\gamma_{v} f_{v}-\rho\left(f_{u}-f_{v}\right) .
\end{aligned}
$$

The function $\bar{\lambda}$ is illustrated in Fig. 7 .

Considering the definition (31) of $\bar{\lambda}$ and the relations (30b) and (30c), one can see that there exists a unique solution $\lambda$ of (30b) if $|\hat{\rho}|<1$. Note that $|\hat{\rho}|<1$ is satisfied if the transmission is both backdrivable and forward-drivable, i.e., $\left|\gamma_{u}\right|<1 \wedge\left|\gamma_{v}\right|<1$. Meanwhile, if $|\hat{\rho}|>1$, the solution $\lambda$ of (30b) may be non-existent or non-unique.

Eliminating $\lambda$ and $s$ from (30) yields the following DI:

$$
(m+M) \dot{v} \in f_{u}-f_{v}-\Phi\left(\operatorname{sgn}(v), \bar{\phi}\left(f_{u}, f_{v}, \hat{\rho}\right), \hat{\rho}\right)
$$

where $\Phi: \mathcal{B} \times \mathbb{R} \times \mathbb{R} \rightrightarrows \mathbb{R}$ is defined as follows:

$$
\Phi(s, \phi, \rho) \triangleq s|\bar{\lambda}(\phi, \rho s)| .
$$

Note that the DI representation (33) is an equivalent form of the proposed DAI representation (14). The DI (33) can be seen as less convenient for physical interpretation but more suited for further mathematical analysis than the DAI (14). If $|\hat{\rho}|<1$, the right-hand side of (33) is single-valued as long as $v \neq 0$. If $|\hat{\rho}|<1$ and $v=0, \Phi(\operatorname{sgn}(v), \phi, \hat{\rho})$ is the closed interval $[\Phi(-1, \phi, \hat{\rho}), \Phi(1, \phi, \hat{\rho})]$ because $\Phi(s, \phi, \hat{\rho})$ is a continuous, monotone, and bounded function of $s \in \mathcal{B}$, as stated in Lemma 2 in Appendix A.
Remark 1 (Existence of solution of the DI (33)). If $|\hat{\rho}|<1$, the right-hand side of (33) is outer semicontinuous ${ }^{2}$ (cf. [18, p. 14]) with respect to $v$, and is compact and convex for all $\left\{v, f_{u}, f_{v}\right\}$. Therefore, according to Theorem 4.7 of [17], the DI (33) has an absolutely continuous solution with respect to $v$ if $f_{u}$ and $f_{v}$ are measurable functions of time $t$.

Remark 2 (Uniqueness of solution of the DI (33)). If $|\hat{\rho}|<1$, the right-hand side of (33) is a locally bounded maximal monotone (cf. [19, Section 2.1.2]) function of $v$. Therefore, according to Theorem 1 in [20], the DI (33) has unique solutions from almost all initial values of $v$.

In the ill-posed case, i.e., in the case of $|\hat{\rho}|>1$, the righthand side of the DI (33) is empty-valued if $\hat{\rho} v \bar{\phi}\left(f_{u}, f_{v}, \hat{\rho}\right)>0$ and dual-valued otherwise. Such cases are often referred to as dynamic jamming or dynamic wedging (see, e.g., Section 3.2 of [21]). One may also say that the rigid-body assumption or the Coulomb-friction assumption fails to hold in this case. Some relevant discussions can be found in [15, Section 5.5.5]. Although a thorough mathematical analysis of this ill-posed system is left outside the scope of the paper, one may interpret the empty-valued case as the situation where the triplet $\left\{f_{u}, f_{v}, v\right\}$ in the DI (33) is not permitted and thus $v$ should instantaneously reach zero with the infinite acceleration $\dot{v}= \pm \infty$. The dual-valued case may be more debatable, but seeing the structure of $\bar{\lambda}$ in Fig. 7, it would be natural to neglect the red parts, which are not continuously connected to other portions of the surface. In this line of consideration, one may replace $\bar{\lambda}$ by the following single-valued function $\bar{\lambda}_{s}: \mathbb{R} \times \mathbb{R} \rightarrow \mathbb{R} \cup\{ \pm \infty\}:$

$$
\bar{\lambda}_{s}(\phi, \rho) \triangleq \begin{cases}0 & \text { if } \phi=0 \\ \phi /(1-\rho) & \text { if } \phi>0 \wedge \rho<1 \\ \phi /(1+\rho) & \text { if } \phi<0 \wedge \rho>-1 \\ \infty & \text { if } \phi>0 \wedge \rho \geq 1 \\ -\infty & \text { if } \phi<0 \wedge \rho \leq-1\end{cases}
$$

which corresponds to the blue surface in Fig. 7, with the empty-valuedness being replaced by $+\infty$ or $-\infty$.

Some researchers [2], [6], [10], [14] describe dynamical systems like Fig. 1 with expressions in which the inertia varies between the forward-driving and backdriving cases. Their results are consistent with the DAI (14) and DI (33). Under the condition $|\hat{\rho}|<1$, the DI (33) reduces to

$$
(m+M) \dot{v}=f_{u}-f_{v}-\bar{\phi}\left(f_{u}, f_{v}, \hat{\rho}\right) /(1-\hat{\rho})
$$

if $v \bar{\phi}\left(f_{u}, f_{v}, \hat{\rho}\right)>0$, and reduces to

$$
(m+M) \dot{v}=f_{u}-f_{v}+\bar{\phi}\left(f_{u}, f_{v}, \hat{\rho}\right) /(1+\hat{\rho})
$$

if $v \bar{\phi}\left(f_{u}, f_{v}, \hat{\rho}\right)<0$. Through some straightforward derivations considering the definitions of $\bar{\phi}, \hat{\rho}, \eta_{f}$, and $\eta_{b}$, one obtains

$$
\left(\eta_{f} m+M\right) \dot{v}=\eta_{f} f_{u}-f_{v}
$$

if $v \bar{\phi}\left(f_{u}, f_{v}, \hat{\rho}\right)>0$, i.e., in the forward-driving case, and

$$
\left(m / \eta_{b}+M\right) \dot{v}=f_{u} / \eta_{b}-f_{v}
$$

\footnotetext{
${ }^{2}$ The outer semicontinuity is often referred to as upper semicontinuity [17, p. 32] in the set-valued sense.
} 
if $v \bar{\phi}\left(f_{u}, f_{v}, \hat{\rho}\right)<0$, i.e., in the backdriving case. These expressions coincide with those in previous work, e.g., [11, Eqs. (18) and (19)], [14, Section II.A], [2, Eqs. (5) and (6)], [10, Eq. (20)], and [6, Eqs. (5) and (6)]. One can say that the presented DI (33) is more convenient in that the internal friction force appears as a single term that does not affect other terms, and in that it stands even in the static friction.

\section{Discrete-time Representation for Simulations}

For simulation purposes, the continuous-time DAI representation (14) or its equivalent DI form (33) needs to be approximated by a discrete-time representation. The explicit discretization, such as the forward Euler scheme, is not applicable because, with such a scheme, the set-valuedness in the DAI (14) or the DI (33) acts only as a discontinuity, which results in chattering around $v=0$ and fails to realize the static friction. This paper applies the implicit (backward) Euler discretization method, which has been known to be useful for the time integration of systems involving Coulomb friction [19], [22]-[25].

The implicit-Euler discretization of the DI (33) can be given as follows:

$$
\begin{aligned}
(m+M) \frac{v_{k}-v_{k-1}}{h} \in & f_{u, k}-f_{v, k} \\
& \quad-\Phi\left(\operatorname{sgn}\left(v_{k}\right), \bar{\phi}\left(f_{u, k}, f_{v, k}, \hat{\rho}\right)\right)
\end{aligned}
$$

where $h$ is the timestep size and the subscript $k$ is the discretetime index. For simulation purposes, (40) must be solved to obtain $v_{k}$ according to given $\left\{v_{k-1}, f_{u, k}, f_{v, k}\right\}$. The algebraic inclusion (40) can be rewritten as follows:

$$
v_{k}-v_{k}^{*} \in-\frac{h \Phi\left(\operatorname{sgn}\left(v_{k}\right), \phi_{k}, \hat{\rho}\right)}{m+M}
$$

where

$$
\begin{aligned}
& v_{k}^{*} \triangleq v_{k-1}+\frac{h\left(f_{u, k}-f_{v, k}\right)}{m+M} \\
& \phi_{k} \triangleq \bar{\phi}\left(f_{u, k}, f_{v, k}, \hat{\rho}\right) .
\end{aligned}
$$

Lemma 2 suggests that the function $\Phi\left(s, \phi_{k}\right)$ is continuous and monotone with respect to $s \in \mathcal{B}$. Therefore, by using the relation (5), one can equivalently rewrite (41) as follows:

$$
v_{k}=v_{k}^{*}-\operatorname{proj}\left(\frac{h \Phi\left(\mathcal{B}, \phi_{k}, \hat{\rho}\right)}{m+M}, v_{k}^{*}\right) \text {. }
$$

Therefore, an algorithm to solve (40) is written as follows:

$$
\begin{aligned}
\phi_{k} & :=\bar{\phi}\left(f_{u, k}, f_{v, k}, \hat{\rho}\right) \\
v_{k}^{*} & :=v_{k-1}+\frac{h\left(f_{u, k}-f_{v, k}\right)}{m+M} \\
v_{k} & :=v_{k}^{*}-\operatorname{proj}\left(\frac{h \Phi\left(\mathcal{B}, \phi_{k}, \hat{\rho}\right)}{m+M}, v_{k}^{*}\right) .
\end{aligned}
$$

The expression (45) is an algorithm of the time integration of the DAI (14) or the DI (33), which updates the velocity $v_{k}$ in accordance with the input forces $f_{u, k}$ and $f_{v, k}$. With the algorithm (45), $v_{k}$ becomes exact zero if

$$
(m+M) v_{k}^{*} / h \in\left[\Phi\left(-1, \phi_{k}, \hat{\rho}\right), \Phi\left(1, \phi_{k}, \hat{\rho}\right)\right],
$$

which means that the algorithm (45) properly captures the static friction. One can use the algorithm (45) even in the illposed case by replacing $\bar{\lambda}$ by $\bar{\lambda}_{s}$ in (35) in which $\infty$ replaced by a very large floating-point number.

\section{Multi-DOF Extension}

\section{A. DAI Representation}

This section considers the equation of motion of an articulated rigid-body system that has $n$ joints driven by actuators through transmissions. First, let us write the equation of motion of an articulated rigid-body system as follows:

$$
\boldsymbol{M}_{h}(\boldsymbol{q}) \ddot{\boldsymbol{q}}+\boldsymbol{h}(\boldsymbol{q}, \dot{\boldsymbol{q}})=\hat{\boldsymbol{\tau}}_{v}+\boldsymbol{J}(\boldsymbol{q})^{T} \boldsymbol{f}
$$

where $\boldsymbol{q} \in \mathbb{R}^{n}$ is the joint angle vector, $\boldsymbol{M}_{h}(\boldsymbol{q}) \in \mathbb{R}^{n \times n}$ is the inertia matrix of the system, $\boldsymbol{h}(\boldsymbol{q}, \dot{\boldsymbol{q}}) \in \mathbb{R}^{n}$ is the force including the centrifugal, Coriolis and gravitational forces, $\boldsymbol{J}(\boldsymbol{q}) \in \mathbb{R}^{n \times 6}$ is the Jacobian matrix that translates the joint velocity to the end-effector velocity, $\boldsymbol{f} \in \mathbb{R}^{6}$ is the external force acting on the end-effector, and $\hat{\boldsymbol{\tau}}_{v} \in \mathbb{R}^{n}$ is the vector of torques applied to the joints.

Next, let us consider the transmissions and the actuators that are to be connected to the system (47). Let $\gamma_{u} \in \mathbb{R}^{n}$ and $\gamma_{v} \in \mathbb{R}^{n}$ be the vectors of the input- and output-side asymmetry coefficients, respectively, $\boldsymbol{m} \in \mathbb{R}^{n}$ and $\boldsymbol{m}_{v} \in \mathbb{R}^{n}$ be the vectors including the input- and output-side moments of inertia of the transmissions, respectively, and $\tau_{u} \in \mathbb{R}^{n}$ be the vector of the actuator torques. Note that $\boldsymbol{m}$ and $\boldsymbol{\tau}_{u}$ are evaluated in the output shafts, i.e, the real moment of inertia of the motors are $\boldsymbol{m}$ divided by the squares of the reduction ratios, and the real actuator torques are $\tau_{u}$ divided by the reduction ratios. Then, the equation of motion of the transmission part of the system can be written as follows:

$$
\begin{aligned}
& \left(\left[\boldsymbol{m}_{r}\right]+\left[\boldsymbol{m}_{v}\right]\right) \ddot{\boldsymbol{q}} \in \boldsymbol{\tau}_{u}-\hat{\boldsymbol{\tau}}_{v}-[|\boldsymbol{\lambda}|] \operatorname{sgn}(\dot{\boldsymbol{q}}) \\
& \left(\left[\boldsymbol{\gamma}_{u}\right][\boldsymbol{m}]-\left[\boldsymbol{\gamma}_{v}\right]\left[\boldsymbol{m}_{v}\right]\right) \ddot{\boldsymbol{q}}=\left[\boldsymbol{\gamma}_{u}\right] \boldsymbol{\tau}_{u}+\left[\boldsymbol{\gamma}_{v}\right] \hat{\boldsymbol{\tau}}_{v}-\boldsymbol{\lambda} .
\end{aligned}
$$

Here, $\lambda \in \mathbb{R}^{n}$ serves as a Lagrange multiplier. Note that (48) is only a collection of $n$ independent systems each of which are in the form of the DAI (14).

By combining the collection (48) of the transmissions with the main part of the articulated rigid-body system (47) and eliminating $\hat{\tau}_{v}$, one obtains the following DAI:

$$
\begin{aligned}
& ([\boldsymbol{m}]+\boldsymbol{M}(\boldsymbol{q})) \ddot{\boldsymbol{q}} \in \boldsymbol{\tau}_{u}-\boldsymbol{\tau}_{v}-[|\boldsymbol{\lambda}|] \operatorname{sgn}(\dot{\boldsymbol{q}}) \\
& \left(\left[\gamma_{u}\right][\boldsymbol{m}]-\left[\gamma_{v}\right] \boldsymbol{M}(\boldsymbol{q})\right) \ddot{\boldsymbol{q}}=\left[\gamma_{u}\right] \boldsymbol{\tau}_{u}+\left[\gamma_{v}\right] \boldsymbol{\tau}_{v}-\boldsymbol{\lambda}
\end{aligned}
$$

where

$$
\begin{aligned}
\boldsymbol{M}(\boldsymbol{q}) & \triangleq \boldsymbol{M}_{h}(\boldsymbol{q})+\left[\boldsymbol{m}_{v}\right] \\
\boldsymbol{\tau}_{v} & \triangleq \boldsymbol{h}(\boldsymbol{q}, \dot{\boldsymbol{q}})-\boldsymbol{J}(\boldsymbol{q})^{T} \boldsymbol{f} .
\end{aligned}
$$

Note that the DAI (49) can be considered as the multi-DOF version of the DAI (14). A mathematical difficulty is raised by the cross-coupling of accelerations resulted from the nondiagonal elements of the matrix $\boldsymbol{M}(\boldsymbol{q})$. 


\section{B. DI Representation}

Let us investigate dynamic properties of the DAI (49) in a similar approach to that in Section IV-B. It can be equivalently rewritten as follows:

$$
\begin{aligned}
& ([\boldsymbol{m}]+\boldsymbol{M}(\boldsymbol{q})) \ddot{\boldsymbol{q}}=\boldsymbol{\tau}_{\alpha}-[\boldsymbol{s}]|\boldsymbol{\lambda}| \\
& \boldsymbol{\lambda}-\boldsymbol{P}(\boldsymbol{q})[\boldsymbol{s}]|\boldsymbol{\lambda}|=\boldsymbol{\tau}_{\lambda}-\boldsymbol{P}(\boldsymbol{q}) \boldsymbol{\tau}_{\alpha} \\
& \boldsymbol{s} \in \operatorname{sgn}(\dot{\boldsymbol{q}})
\end{aligned}
$$

where

$$
\begin{aligned}
\boldsymbol{P}(\boldsymbol{q}) & \triangleq\left(\left[\boldsymbol{\gamma}_{u}\right][\boldsymbol{m}]-\left[\boldsymbol{\gamma}_{v}\right] \boldsymbol{M}(\boldsymbol{q})\right)([\boldsymbol{m}]+\boldsymbol{M}(\boldsymbol{q}))^{-1} \\
\boldsymbol{\tau}_{\alpha} & \triangleq \boldsymbol{\tau}_{u}-\boldsymbol{\tau}_{v} \\
\boldsymbol{\tau}_{\lambda} & \triangleq\left[\boldsymbol{\gamma}_{u}\right] \boldsymbol{\tau}_{u}+\left[\boldsymbol{\gamma}_{v}\right] \boldsymbol{\tau}_{v} .
\end{aligned}
$$

Note that $\boldsymbol{P}(\boldsymbol{q})$ can be seen as a multi-dimensional version of the coefficient $\hat{\rho}$ defined in (29). Let us define a function $\bar{\lambda}: \mathbb{R}^{n} \times \mathbb{R}^{n \times n} \rightrightarrows \mathbb{R}^{n}$ as follows:

$$
\overline{\boldsymbol{\lambda}}(\boldsymbol{\phi}, \boldsymbol{P}) \triangleq\left\{\boldsymbol{\lambda} \in \mathbb{R}^{n}|\boldsymbol{\lambda}-\boldsymbol{P}| \boldsymbol{\lambda} \mid=\boldsymbol{\phi}\right\} .
$$

Then, (52) can be rewritten as follows:

$$
\begin{aligned}
& ([\boldsymbol{m}]+\boldsymbol{M}(\boldsymbol{q})) \ddot{\boldsymbol{q}}=\boldsymbol{\tau}_{\alpha}-[|\boldsymbol{\lambda}|] \boldsymbol{s} \\
& \boldsymbol{\lambda} \in \overline{\boldsymbol{\lambda}}\left(\boldsymbol{\tau}_{\lambda}-\boldsymbol{P}(\boldsymbol{q}) \boldsymbol{\tau}_{\alpha}, \boldsymbol{P}(\boldsymbol{q})[\boldsymbol{s}]\right) \\
& \boldsymbol{s} \in \operatorname{sgn}(\dot{\boldsymbol{q}}) .
\end{aligned}
$$

Note that $\bar{\lambda}$ in (56) reduces to $\bar{\lambda}$ in (31) with $n=1$. Lemma 3 in Appendix A states that $\overline{\boldsymbol{\lambda}}(\boldsymbol{\phi}, \boldsymbol{P}[s])$ is singlevalued if $\sigma_{\max }(\boldsymbol{P}[\boldsymbol{s}])<1$. Considering that $\sigma_{\max }([\boldsymbol{s}]) \leq 1$, $\sigma_{\max }(\boldsymbol{P}(\boldsymbol{q}))<1$ is sufficient for the uniqueness of $\boldsymbol{\lambda}$ in $(57 \mathrm{~b})$. Moreover, the proof of Lemma 3, which uses the fixed point theorem, suggests that $\bar{\lambda}(\phi, P)$ can be numerically obtained by several iterations of the computation $\lambda:=\phi+P|\lambda|$ with the initial value setting $\boldsymbol{\lambda}:=\phi$.

Unfortunately, the condition $\sigma_{\max }(\boldsymbol{P}(\boldsymbol{q}))<1$ may be violated even if all joints are both backdrivable and forwarddrivable. One can see that all joints are backdrivable (resp., forward-drivable) if and only if all the diagonal elements of $\left[\gamma_{v}\right]$ (resp., $\left[\gamma_{u}\right]$ ) are smaller than 1. Therefore, all joints of the system are both backdrivable and forward-drivable if $\sigma_{\max }\left(\left[\gamma_{u}\right]\right)<1 \wedge \sigma_{\max }\left(\left[\gamma_{v}\right]\right)<1$. This condition is not sufficient for the condition $\sigma_{\max }(\boldsymbol{P}(\boldsymbol{q}))<1$. A clear physical interpretation for the condition $\sigma_{\max }(\boldsymbol{P}(\boldsymbol{q}))<1$ is left as an open problem.

By eliminating $\boldsymbol{\lambda}$ and $s$ from (57), one can obtain the following DI representation:

$$
\begin{aligned}
& ([\boldsymbol{m}]+\boldsymbol{M}(\boldsymbol{q})) \ddot{\boldsymbol{q}} \in \boldsymbol{\tau}_{\alpha} \\
& \quad-\boldsymbol{\Phi}\left(\operatorname{sgn}(\dot{\boldsymbol{q}}), \boldsymbol{\tau}_{\lambda}-\boldsymbol{P}(\boldsymbol{q}) \boldsymbol{\tau}_{\alpha}, \boldsymbol{P}(\boldsymbol{q})\right)
\end{aligned}
$$

where $\mathbf{\Phi}: \mathcal{B}^{n} \times \mathbb{R}^{n} \times \mathbb{R}^{n \times n} \rightrightarrows \mathbb{R}^{n}$ is defined as follows:

$$
\boldsymbol{\Phi}(s, \boldsymbol{\phi}, \boldsymbol{P}) \triangleq[\boldsymbol{s}]|\overline{\boldsymbol{\lambda}}(\boldsymbol{\phi}, \boldsymbol{P}[\boldsymbol{s}])| .
$$

The DI representation (58) is a multi-dimensional version of the DI (33), and $\boldsymbol{\Phi}$ in (59) is a multi-dimensional version of $\Phi$ in (34). As long as $\sigma_{\max }(\boldsymbol{P})<1, \boldsymbol{\Phi}(\boldsymbol{s}, \boldsymbol{\phi}, \boldsymbol{P})$ is single-valued because of the property of $\bar{\lambda}$ stated in Lemma 3 . Under this condition, the right-hand side of (58) is set-valued only if $\dot{\boldsymbol{q}}$ has one or more zero elements.
Remark 3 (Solution of the DI (58)). For the existence of the uniqueness of solution of the DI (58), the continuity and the monotonicity of the map from $s$ to $\bar{\lambda}(\phi, P[s])$ are important. Under the condition $\sigma_{\max }(\boldsymbol{P})<1$, its continuity is implied by Lemma 4, but its monotonicity, unfortunately, has not been proven yet. The continuity implies that the right-hand side of (58) is outer semicontinuous (cf. [18, p. 14]) with respect to $\dot{\boldsymbol{q}}$. If the monotonicity of $\overline{\boldsymbol{\lambda}}(\boldsymbol{\phi}, \boldsymbol{P}[\boldsymbol{s}])$ is proven, the righthand side can be shown to be compact and convex for all $\left\{\boldsymbol{q}, \dot{\boldsymbol{q}}, \boldsymbol{\tau}_{u}, \boldsymbol{f}\right\}$. Then, Theorem 4.7 of [17] suggests that the DI (58) always has an absolutely continuous solution with respect to $\{\boldsymbol{q}, \dot{\boldsymbol{q}}\}$ if $\boldsymbol{\tau}_{u}$ and $\boldsymbol{f}$ are measurable functions of time $t$. In addition, if the monotonicity of $\overline{\boldsymbol{\lambda}}(\boldsymbol{\phi}, \boldsymbol{P}[\boldsymbol{s}])$ is proven, the right-hand side of (58) can be shown to be a locally bounded maximal monotone function of $\{\boldsymbol{q}, \dot{\boldsymbol{q}}\}$. In this case, Theorem 1 in [20] suggests that the DI (58) has unique solutions from almost all initial values of $\{\boldsymbol{q}, \dot{\boldsymbol{q}}\}$.

\section{Discrete-time Representation for Simulations}

A simulation algorithm for the time integration of the DAI (49) or the DI (58) is now derived. An implicit-Euler discretization of the DI (58) can be given as follows:

$$
\begin{aligned}
& \hat{\boldsymbol{M}}_{k-1} \frac{\boldsymbol{v}_{k}-\boldsymbol{v}_{k-1}}{h} \in \boldsymbol{\tau}_{\alpha, k}-\boldsymbol{\Phi}\left(\operatorname{sgn}\left(\boldsymbol{v}_{k}\right), \phi_{k}, \boldsymbol{P}_{k-1}\right) \\
& \boldsymbol{q}_{k}=\boldsymbol{q}_{k-1}+h \boldsymbol{v}_{k}
\end{aligned}
$$

where

$$
\begin{aligned}
\boldsymbol{\phi}_{k} & \triangleq \boldsymbol{\tau}_{\lambda, k}-\boldsymbol{P}_{k-1} \boldsymbol{\tau}_{\alpha, k} \\
\hat{\boldsymbol{M}}_{k-1} & \triangleq[\boldsymbol{m}]+\boldsymbol{M}\left(\boldsymbol{q}_{k-1}\right) \\
\boldsymbol{P}_{k-1} & \triangleq \boldsymbol{P}\left(\boldsymbol{q}_{k-1}\right) .
\end{aligned}
$$

The algebraic inclusion (60) is rewritten as follows:

$$
\boldsymbol{v}_{k}^{*}-\boldsymbol{v}_{k} \in h \hat{M}_{k-1}^{-1} \boldsymbol{\Phi}\left(\operatorname{sgn}\left(\boldsymbol{v}_{k}\right), \phi_{k}, \boldsymbol{P}_{k-1}\right)
$$

where

$$
\boldsymbol{v}_{k}^{*} \triangleq \boldsymbol{v}_{k-1}+h \hat{\boldsymbol{M}}_{k-1}^{-1} \boldsymbol{f}_{\alpha, k} .
$$

One needs to obtain $\boldsymbol{v}_{k}$ by solving (65). To this end, let us define an operator axproj: $\mathbb{R}^{n} \times \mathbb{R}^{n \times n} \times \mathbb{R}^{n} \times \mathbb{R}^{n \times n} \rightarrow \mathbb{R}^{n}$ as follows:

$$
\begin{aligned}
& \underset{\operatorname{axproj}}{ }(\boldsymbol{v}, \boldsymbol{A}, \boldsymbol{\phi}, \boldsymbol{P}) \\
& \quad \triangleq\left\{\boldsymbol{y} \in \mathbb{R}^{n} \mid \boldsymbol{y} \in \boldsymbol{A} \boldsymbol{\Phi}(\operatorname{sgn}(\boldsymbol{v}-\boldsymbol{y}), \boldsymbol{\phi}, \boldsymbol{P})\right\} .
\end{aligned}
$$

Then, the solution $\boldsymbol{v}_{k}$ of (65) is written as follows:

$$
\boldsymbol{v}_{k}=\boldsymbol{v}_{k}^{*}-\operatorname{axproj}\left(\boldsymbol{v}_{k}^{*}, h \boldsymbol{M}_{k-1}^{-1}, \phi_{k}, \boldsymbol{P}_{k-1}\right) .
$$

Provided that an algorirhm for the operator axproj is available, one can obtain an algorithm for the time integration of the DAI (49) or the DI (58) as follows:

$$
\begin{aligned}
\hat{\boldsymbol{M}}_{k-1} & :=[\boldsymbol{m}]+\boldsymbol{M}\left(\boldsymbol{q}_{k-1}\right) \\
\boldsymbol{P}_{k-1} & :=\left(\left[\boldsymbol{\gamma}_{u}\right][\boldsymbol{m}]-\left[\boldsymbol{\gamma}_{v}\right] \boldsymbol{M}\left(\boldsymbol{q}_{k-1}\right)\right) \hat{\boldsymbol{M}}_{k-1}^{-1} \\
\boldsymbol{\tau}_{v, k} & :=\boldsymbol{h}\left(\boldsymbol{q}_{k-1}, \boldsymbol{v}_{k-1}\right)-\boldsymbol{J}\left(\boldsymbol{q}_{k-1}\right)^{T} \boldsymbol{f}_{k} \\
\boldsymbol{\phi}_{k} & :=\left[\boldsymbol{\gamma}_{u}\right] \boldsymbol{\tau}_{u, k}+\left[\boldsymbol{\gamma}_{v}\right] \boldsymbol{\tau}_{v, k}-\boldsymbol{P}_{k-1}\left(\boldsymbol{\tau}_{u, k}-\boldsymbol{\tau}_{v, k}\right) \\
\boldsymbol{v}_{k}^{*} & :=\boldsymbol{v}_{k-1}+h \hat{\boldsymbol{M}}_{k-1}^{-1}\left(\boldsymbol{\tau}_{u, k}-\boldsymbol{\tau}_{v, k}\right)
\end{aligned}
$$




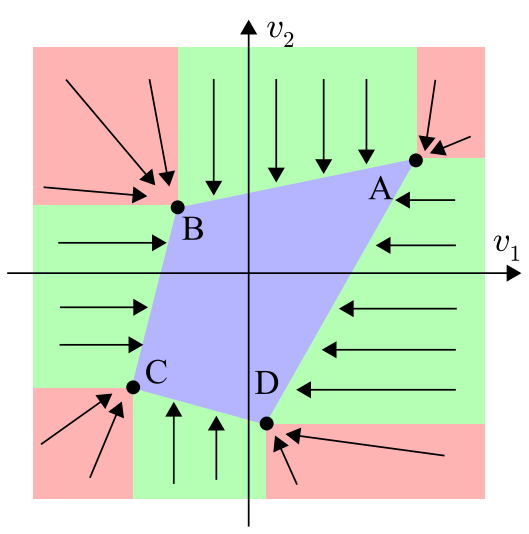

Fig. 8. An illustration of the operator axproj defined in (67) with $n=2$. The vertices A, B, C, and D are $\boldsymbol{A} \boldsymbol{\Phi}(\boldsymbol{s}, \boldsymbol{\phi}, \boldsymbol{P})$ where $\boldsymbol{s}=[1,1]^{T},[-1,1]^{T}$, $[-1,-1]^{T}$, and $[1,-1]^{T}$, respectively. When $\boldsymbol{v}$ is in the pink regions, $\boldsymbol{y}=$ $\operatorname{axproj}(\boldsymbol{v}, \boldsymbol{A}, \boldsymbol{\phi}, \boldsymbol{P})$ is the closest vertex. When $\boldsymbol{v}$ is in the green regions, $\boldsymbol{y}$ is the projection of $\boldsymbol{v}$ onto the closest edge along either axis. When $\boldsymbol{v}$ is within the quadrilateral $\mathrm{ABCD}, \boldsymbol{y}=\boldsymbol{v}$.

$$
\begin{aligned}
& \boldsymbol{v}_{k}:=\boldsymbol{v}_{k}^{*}-\operatorname{axproj}\left(\boldsymbol{v}_{k}^{*}, h \hat{\boldsymbol{M}}_{k-1}^{-1}, \phi_{k}, \boldsymbol{P}_{k-1}\right) \\
& \boldsymbol{q}_{k}:=\boldsymbol{q}_{k-1}+h \boldsymbol{v}_{k} .
\end{aligned}
$$

Fig. 8 illustrates how the operator axproj should work. Here, $\boldsymbol{A} \boldsymbol{\Phi}\left(\mathcal{B}^{n}, \boldsymbol{\phi}, \boldsymbol{P}\right)$ is a polyhedron spanned by $2^{n}$ vertices in $\mathbb{R}^{n}$, and $\boldsymbol{y}=\operatorname{axproj}(\boldsymbol{v}, \boldsymbol{A}, \boldsymbol{\phi}, \boldsymbol{P})$ is the projection of $\boldsymbol{v}$ on the polyhedron in a particular axis-aligned manner. Note that (68) is a multi-dimensional version of (44). As a special case, if both $\boldsymbol{A}$ and $\boldsymbol{P}$ are diagonal matrices, the computation of axproj reduces to the following:

$$
\operatorname{axproj}(\boldsymbol{v}, \boldsymbol{A}, \boldsymbol{\phi}, \boldsymbol{P})=\left[\begin{array}{c}
\operatorname{proj}\left(A_{11} \Phi\left(\mathcal{B}, \phi_{1}, P_{11}\right), v_{1}\right) \\
\vdots \\
\operatorname{proj}\left(A_{n n} \Phi\left(\mathcal{B}, \phi_{n}, P_{n n}\right), v_{n}\right)
\end{array}\right]
$$

where the symbols with subscripts denote elements of correspondent vectors or matrices.

There are several ways to perform the computation of axproj. An important fact is that it is classified as a variational inequality (VI) problem [26, Section 1.1]. By borrowing the notation of [26, Section 1.1], a VI problem is a problem to obtain $x \in \mathbb{R}^{n}$ for a convex set $K \subset \mathbb{R}^{n}$ and a map $F: \mathbb{R}^{n} \rightarrow \mathbb{R}^{n}$ so that

$$
x \in \operatorname{SOL}(K, F) \triangleq\left\{x \in \mathbb{R}^{n} \mid 0 \in F(x)+\mathcal{N}(x, K)\right\}
$$

where $\mathcal{N}$ is an operator called the normal cone (see [26, Section 1.1] for its definition). One can see that, if one defines a function $F: \mathbb{R}^{n} \rightarrow \mathbb{R}^{n}$ as

$$
F(s) \triangleq A \Phi(s, \phi, P)-\boldsymbol{v},
$$

the operator axproj can be rewritten as follows:

$$
\operatorname{axproj}(\boldsymbol{v}, \boldsymbol{A}, \boldsymbol{\phi}, \boldsymbol{X})=F\left(\operatorname{SOL}\left(\mathcal{B}^{n}, F\right)\right)+\boldsymbol{v} .
$$

One simple way to solve a VI is, as stated in $[19$, Section 12.6.7], to perform an iteration of $s:=\operatorname{proj}(K, s-$ $c F(s))$ with a small positive number $c$ and an appropriate initial value of $s$. After obtaining the solution $s$ of the VI, the output $\boldsymbol{y}$ of axproj can be obtained by $\boldsymbol{y}:=F(\boldsymbol{s})+\boldsymbol{v}$.

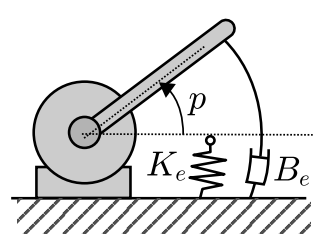

(a)

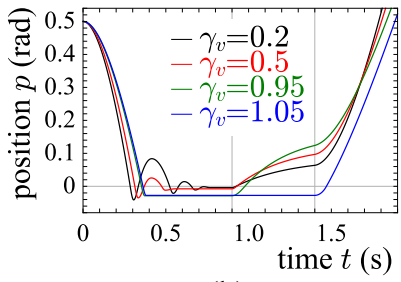

(b)

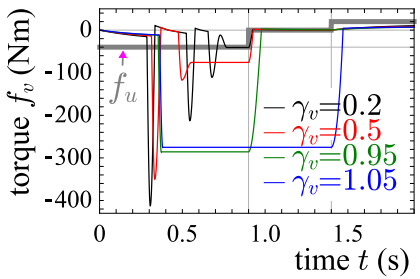

(c)
Fig. 9. One-DOF simulation; (a) the system, (b) results of position $p$, and (c) results of force $f_{v}$.

More computationally efficient schemes may be found in the literature, e.g., [19], [26], or can be constructed considering the particular structure of the function $F$ in (72), but it is left outside the scope of this paper. It should also be noted that, if $\boldsymbol{P}=0$, it reduces to an affine variational inequality (AVI) problem, for which more sophisticated solvers are available.

\section{NUMERICAL EXAMPLES}

This section presents illustrative simulation results of the proposed algorithms (45) and (69). The paper does not attempt to compare them with previous methods because, as far as the author is aware, there are no existing models that capture asymmetric friction with the static friction.

\section{A. 1-DOF System}

Some simulations of a 1-DOF system are performed to validate the algorithm (45). The system is illustrated in Fig. 9(a), in which a rod is driven by a rotary actuator through a transmission. The timestep size is set as $h=0.001 \mathrm{~s}$. The parameters are set as $m=1 \mathrm{~kg} \cdot \mathrm{m}^{2}, M=1 \mathrm{~kg} \cdot \mathrm{m}^{2}$, $\gamma_{u}=0.2$, and $\gamma_{v} \in\{0.2,0.5,0.95,1.05\}$. The parameter $M$ includes the moment of inertia of the rod. As illustrated in Fig. 9(a), the rod is always subject to the viscosity $B_{e}=$ $10 \mathrm{Nms} / \mathrm{rad}$, and gains contact with an external object with the stiffness $K_{e}=10000 \mathrm{Nm} / \mathrm{rad}$ at the location $p=0 \mathrm{rad}$. Thus, the force from the rod to the external environment is $f_{v}=\min \left(K_{e} p, 0\right)+B_{e} \dot{p}$. The actuator force $f_{u}$ is set as $f_{u}=-40 \mathrm{Nm}$ in the beginning, switched to $f_{u}=0 \mathrm{Nm}$ at $t=0.9 \mathrm{~s}$, and to $f_{u}=20 \mathrm{Nm}$ at $t=1.4 \mathrm{~s}$.

Note that $\gamma_{v}>1$ means that the transmission is nonbackdrivable. The value of $\hat{\rho}$, defined in (29), for the aforementioned four values of $\gamma_{v}$ satisfy $|\hat{\rho}|<1$ and thus the system is well-posed for all cases. It should also be noted that the reduction ratio $\kappa$ does not appear in this system because the aforementioned values of $m$ and $f_{u}$ are those evaluated at the output shaft; i.e., being evaluated at the input shaft, the actuator's moment of inertia is $m / \kappa^{2}$ and the torque generated by the actuator is $f_{u} / \kappa$. 


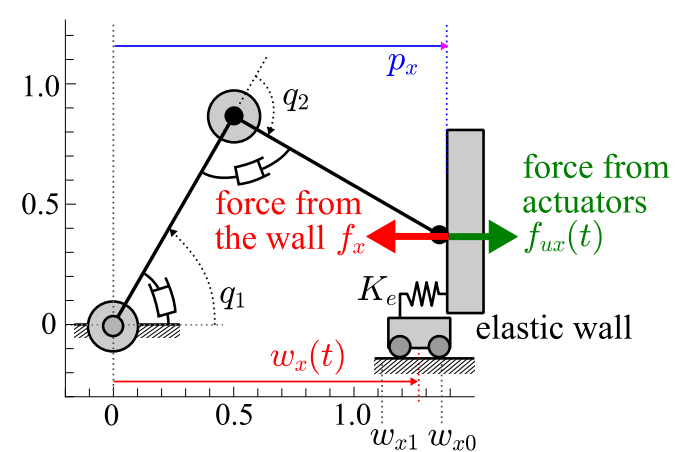

Fig. 10. Two-DOF simulation. The actuator force $\boldsymbol{\tau}_{u}=\boldsymbol{J}(\boldsymbol{q})\left[f_{u x}(t), 0\right]^{T}$ and the wall position $w_{x}(t)$ are given as functions of time $t$.

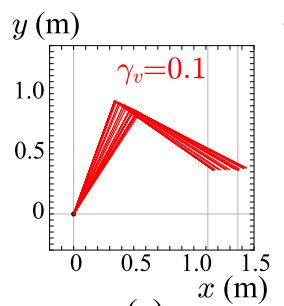

(a)

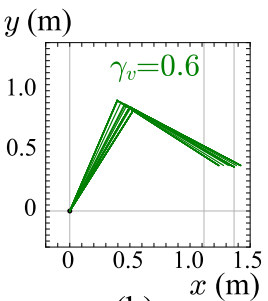

(b)

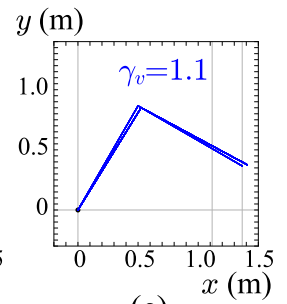

(c)

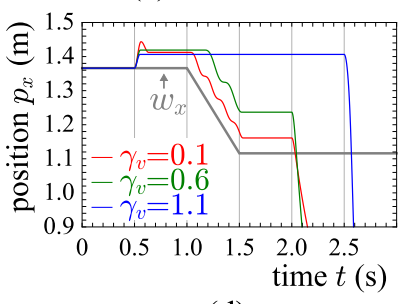

(d)

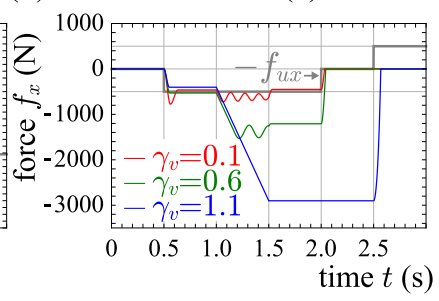

(e)
Fig. 11. Results of two-DOF simulations with $\gamma_{v}=[0.1,0.1]^{T},[0.6,0.6]^{T}$, and $[1.1,1.1]^{T} ;$ (a)(b)(c) snapshots at $0.3 \mathrm{~s}$ intervals for $t<2.0 \mathrm{~s}$, (e) the position $p_{x}$ of the robot's end-effector, and (e) the force $f_{x}$ from the wall to the robot.

Results are shown in Figs. 9(b) and (c). In the beginning, the rod is moved in the negative direction until the collision with the spring because of $f_{u}=-40 \mathrm{Nm}$. The rod is then pushed against the spring until $t=0.9 \mathrm{~s}$ with the force $f_{u}=-40 \mathrm{Nm}$. During this time, the rod bounces on the spring with smaller $\gamma_{v}$ values, but maintains large contact force $f_{v}$, much larger than the actuator force $f_{u}$, with larger $\gamma_{v}$ values. These results properly reflect the asymmetric friction of the transmission.

Once $f_{u}$ is set zero at $t=0.9 \mathrm{~s}$, the rod is pushed back from the spring with $\left|\gamma_{v}\right|<1$, but it is still stuck with $\left|\gamma_{v}\right|>1$. In this period, the contact force $f_{v}$ is still large with $\left|\gamma_{v}\right|>1$ even though the actuator force $f_{u}$ is zero. It is consistent with the fact that the transmission is non-backdrivable with $\left|\gamma_{v}\right|>$ 1. After the actuator force $f_{u}$ is switched to be positive at $t=1.4 \mathrm{~s}$ to pull the rod in the positive direction, the rod is detached also with $\left|\gamma_{v}\right|>1$. These results properly reflect the asymmetric friction behaviors of transmissions, both those backdrivable and non-backdrivable.

\section{B. 2-DOF System}

Some simulations are also performed with a 2-DOF system to validate the algorithm (69). The timestep size is set as

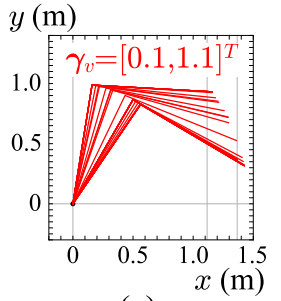

(a)

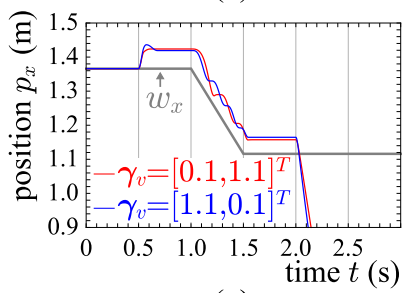

(c)

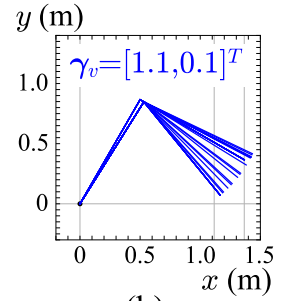

(b)

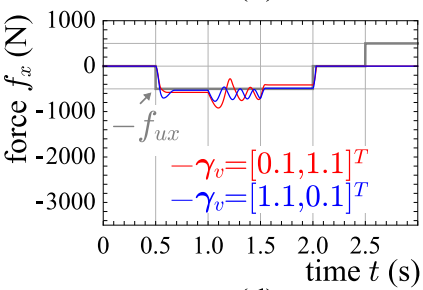

(d)

Fig. 12. Results of two-DOF simulations with $\gamma_{v}=[0.1,1.1]^{T}$ and $[1.1,0.1]^{T}$; (a)(b) snapshots at $0.3 \mathrm{~s}$ intervals for $t<2.0 \mathrm{~s}$, (c) the position $p_{x}$ of the robot's end-effector, and (d) the force $f_{x}$ from the wall to the robot.

$h=0.001 \mathrm{~s}$. The system is a two-link robot driven by rotary actuators through transmissions as in Fig. 10. Each link has a length of $1 \mathrm{~m}$ and a mass of $1 \mathrm{~kg}$ lumped at its distal end. The rotor inertias of the actuators evaluated at the output shafts are both $m=1 \mathrm{~kg} \cdot \mathrm{m}^{2}$. Each joint is subject to the viscosity of $5 \mathrm{Nms} / \mathrm{rad}$. The gravity is assumed not to act. As illustrated in Fig. 10, an elastic wall is placed in parallel to the $y$ axis and its $x$-coordinate $w_{x}$ is moved from the initial position $w_{x 0} \approx 1.37 \mathrm{~m}$ to the terminal position $w_{x 1} \approx 1.12 \mathrm{~m}$. The wall applies the force $\boldsymbol{f}=\left[-K \max \left(p_{x}-w_{x}\right), 0\right]^{T}$ to the end-effector of the robot.

The actuators' torques $\tau_{u} \in \mathbb{R}^{2}$ are determined by an $x$-direction force command $f_{u x}$ through the statics $\tau_{u}:=$ $\boldsymbol{J}(\boldsymbol{q})^{T}\left[f_{u x}, 0\right]^{T}$ where $\boldsymbol{J}(\boldsymbol{q}) \in \mathbb{R}^{2 \times 2}$ is the Jacobian matrix translating the joint velocities to the end-effector velocity. At $t=0$, the end-effector's $x$-coordinate is set to be the same as the initial position of the wall $w_{x 0}$. The simulation scenario is defined as follows:

- $t \in[0 \mathrm{~s}, 0.5 \mathrm{~s}] ; f_{u x}:=0 \mathrm{~N}, w_{x}:=w_{x 0}$; The robot is in contact with the wall.

- $t \in[0.5 \mathrm{~s}, 1 \mathrm{~s}] ; f_{u x}:=500 \mathrm{~N}, w_{x}:=w_{x 0}$; The robot pushes the wall.

- $t \in[1 \mathrm{~s}, 1.5 \mathrm{~s}] ; f_{u x}:=500 \mathrm{~N}, w_{x}:=\left((1.5-t) w_{x 0}+(t-\right.$ 1) $\left.w_{x 1}\right) / 0.5$; The wall moves to the left, pushing back the robot.

- $t \in[1.5 \mathrm{~s}, 2 \mathrm{~s}] ; f_{u x}:=500 \mathrm{~N}, w_{x}:=w_{x 1}$ The wall stops, while the robot keeps pushing.

- $t \in[2 \mathrm{~s}, 2.5 \mathrm{~s}] ; f_{u x}:=0 \mathrm{~N}, w_{x}:=w_{x 1}$; The robot stops pushing.

- $t \in[2.5 \mathrm{~s}, 3 \mathrm{~s}] ; f_{u x}:=-500 \mathrm{~N}, w_{x}:=w_{x 1}$; The robot pulls itself apart from the wall.

Simulations are performed with $\gamma_{u}=[0.1,0.1]^{T}$ and three different settings for $\gamma_{v}$, which are $\gamma_{v}=[0.1,0.1]^{T}$, $[0.6,0.6]^{T}$, and $[1.1,1.1]^{T}$. The results are shown in Fig. 11 . It shows that a larger value of $\gamma_{v}$ results in a large contact force $f_{x}$ when the wall pushes the robot to the left $(t \in[1 \mathrm{~s}, 2 \mathrm{~s}])$. 
In addition, in the case of $\left|\gamma_{v}\right|>1$, the robot is not moved by the wall $(t \in[1 \mathrm{~s}, 1.5 \mathrm{~s}])$ and the contact force is high even after the robot stops pushing ( $t \in[2.0 \mathrm{~s}, 2.5 \mathrm{~s}])$, exhibiting the non-backdrivability of the mechanism. Even in this case, the end-effector is pulled apart from the wall when $f_{u x}$ is set negative, exhibiting the forward-drivability of the system. These results indicate that the presented algorithm (69) is capable of properly simulating the transmission dynamics, including backdrivability and forward-drivability.

Another set of simulations with the same scenario is performed with different joint properties; $\gamma_{v}=[0.1,1,1]^{T}$ and $\gamma_{v}=[1.1,0.1]^{T}$. The results are shown in Fig. 12. It shows that, when the wall pushes the robot to the left $(t \in[1 \mathrm{~s}, 1.5 \mathrm{~s}])$, the joint with $\left|\gamma_{v}\right|>1$ is fixed while the other joint is moved. This result is physically plausible, exhibiting the validity of the presented algorithm (69).

\section{CONCLUding REMARKS}

This paper has presented a dynamics modeling scheme for gear transmissions having asymmetric and load-dependent friction. The representation is of the form of a DAI composed of a 'visible' dynamics and a 'hidden' dynamics combined through a Lagrange multiplier. The presented model properly captures the static friction and even the non-backdrivability. It is characterized by two coefficients that have simple relations with the forward and backward efficiencies of the transmission. The model is extended into a multi-dimensional model for robotic systems equipped with joint transmissions. Discrete-time simulation algorithms are derived through the implicit-Euler discretization of the presented DAIs. Some illustrative simulation results are presented.

The presented model would be further improved by including the load-independent component of Coulomb-like friction and the Stribeck-like effect caused by the lubricants in the transmission. An efficient calibration method for the parameters $\gamma_{u}$ and $\gamma_{v}$ would also need to be sought. Moreover, the control applications of the method, such as friction compensation, would demand some clarifications and improvements on the computational efficiency of the algorithm axproj.

\section{APPENDIX A \\ SOME LEMMAS}

Lemma 1 (About $\bar{\lambda}$ ). For any sets of real numbers $\{\rho, \phi, \lambda\}$, the following statement holds true:

$$
\lambda-\rho|\lambda|=\phi \Longleftrightarrow \lambda \in \bar{\lambda}(\rho, \phi)
$$

where $\bar{\lambda}: \mathbb{R} \times \mathbb{R} \rightrightarrows \mathbb{R}$ is defined in (31).

Proof. If $\lambda=0$, the statement (74) reduces to

$$
\phi=0 \Longleftrightarrow 0 \in \bar{\lambda}(\rho, \phi),
$$

which can be seen to be true by definition (31) of $\bar{\lambda}$.

If $\lambda \neq 0$, one has the following:

$$
\begin{aligned}
& \lambda-\rho|\lambda|=\phi \\
& \Longleftrightarrow \lambda=\phi /(1-\rho \operatorname{sgn}(\lambda)) \\
& \Longleftrightarrow(\lambda=\phi /(1-\rho)>0) \vee(\lambda=\phi /(1+\rho)<0)
\end{aligned}
$$

$$
\begin{aligned}
\Longleftrightarrow & (\lambda=\phi /(1-\rho) \wedge \phi<0 \wedge \rho>1) \\
& \vee(\lambda=\phi /(1-\rho) \wedge \phi>0 \wedge \rho<1) \\
& \vee(\lambda=\phi /(1+\rho) \wedge \phi>0 \wedge \rho<-1) \\
& \vee(\lambda=\phi /(1+\rho) \wedge \phi<0 \wedge \rho>-1) \\
\Longleftrightarrow & (\lambda \in\{\phi /(1-\rho), \phi /(1+\rho)\} \wedge \phi>0 \wedge \rho<-1) \\
& \vee(\lambda \in\{\phi /(1-\rho), \phi /(1+\rho)\} \wedge \phi<0 \wedge \rho>1) \\
& \vee(\lambda=\phi /(1-\rho) \wedge \phi>0 \wedge \rho \in[-1,1)) \\
& \vee(\lambda=\phi /(1+\rho) \wedge \phi<0 \wedge \rho \in(-1,1]) \\
\Longleftrightarrow & \lambda \in \bar{\lambda}(\rho, \phi),
\end{aligned}
$$

which demonstrates (74).

Lemma 2 (Continuity, boundedness, and monotonicity of $\Phi$ ). For all $\{s, \phi, \rho\} \in \mathcal{B} \times \mathbb{R} \times \mathbb{R}$ satisfying $|\rho|<1$, the function $\Phi(s, \phi, \rho)$ of $s$ defined in (34) is continuous, bounded, and monotone.

Proof. The continuity and boundedness are trivial because of the definition (34) of $\Phi$, which depends on the definition (31) of $\bar{\lambda}$. The monotonicity is proven as follows. First, if $\rho=0$, $\bar{\lambda}(\phi, \rho s)=\phi$ and $\Phi(s, \phi, \rho)=s|\phi|$, which is monotone with respect to $s$. Next, if $\rho \neq 0$, Lemma 1 suggests that

$$
\bar{\lambda}(\phi, \rho s)-\rho s|\bar{\lambda}(\phi, \rho s)|=\phi
$$

and, because of the definition (34) of $\Phi$, one has

$$
\Phi(s, \phi, \rho)=(\bar{\lambda}(\phi, \rho s)-\phi) / \rho .
$$

The definition (31) implies that the right-hand side of (78) is monotone with respect to $s$ in both cases of $\rho>0$ and $\rho<0$.

Lemma 3 (Single-valuedness of $\overline{\boldsymbol{\lambda}}$ ). For all $\{\boldsymbol{\phi}, \boldsymbol{P}\} \in \mathbb{R}^{n} \times$ $\mathbb{R}^{n \times n}$ satisfying $\sigma_{\max }(\boldsymbol{P})<1$, the set $\overline{\boldsymbol{\lambda}}(\boldsymbol{\phi}, \boldsymbol{P})$ is a singleton where $\bar{\lambda}: \mathbb{R}^{n} \times \mathbb{R}^{n \times n} \rightrightarrows \mathbb{R}^{n}$ is defined in (56).

Proof. Let $\mathcal{X}(\boldsymbol{\lambda}) \triangleq \boldsymbol{P}|\boldsymbol{\lambda}|+\boldsymbol{\phi}$ and assume that $\sigma_{\max }(\boldsymbol{P})<1$. Then, the map $\mathcal{X}$ is contracting, i.e., $\forall\{\boldsymbol{x}, \boldsymbol{y}\} \in \mathbb{R}^{n} \times \mathbb{R}^{n}$,

$$
\begin{aligned}
& \|\mathcal{X}(\boldsymbol{x})-\mathcal{X}(\boldsymbol{y})\|=\|\boldsymbol{P}(|\boldsymbol{x}|-|\boldsymbol{y}|)\| \\
& \quad \leq \sigma_{\max }(\boldsymbol{P})\||\boldsymbol{x}|-|\boldsymbol{y}|\|<\||\boldsymbol{x}|-|\boldsymbol{y}|\| \leq\|\boldsymbol{x}-\boldsymbol{y}\| .
\end{aligned}
$$

Thus, due to Banach fixed-point theorem, there exists a unique solution for $\mathcal{X}(\boldsymbol{\lambda})=\boldsymbol{\lambda}$, and it means that the inclusion $\boldsymbol{\lambda} \in$ $\overline{\boldsymbol{\lambda}}(\phi, P)$ has a unique solution.

Remark 4. The proof of Lemma 3 is along the same line as is the proof of Proposition 9 of [21], which deals with a system that is almost the same as (49).

Lemma 4 (Continuity and boundedness of $\bar{\lambda}$ ). For all $\{\boldsymbol{\phi}, \boldsymbol{P}, \boldsymbol{s}\} \in \mathbb{R}^{n} \times \mathbb{R}^{n \times n} \times \mathcal{B}^{n}$ satisfying $\sigma_{\max }(\boldsymbol{P})<1$, the single-valued function $\overline{\boldsymbol{\lambda}}(\boldsymbol{\phi}, \boldsymbol{P}[\boldsymbol{s}])$ of $\boldsymbol{s}$ is continuous and bounded.

Proof. For brevity, let us use $\sigma_{P}=\sigma_{\max }(\boldsymbol{P})<1$. With $s \in$ $\mathcal{B}^{n}$ and $\boldsymbol{\lambda}=\overline{\boldsymbol{\lambda}}(\boldsymbol{\phi}, \boldsymbol{P}[\boldsymbol{s}])$, one has the following,

$$
\|\boldsymbol{\phi}\|=\|\boldsymbol{\lambda}-\boldsymbol{P}[\boldsymbol{s}]|\boldsymbol{\lambda}|\| \geq\left(1-\sigma_{P}\|\boldsymbol{s}\|_{\infty}\right)\|\boldsymbol{\lambda}\|,
$$

which results in the following:

$$
\|\boldsymbol{\lambda}\|_{\infty} \leq\|\boldsymbol{\lambda}\| \leq \frac{\|\boldsymbol{\phi}\|}{1-\sigma_{P}\|s\|_{\infty}} \leq \frac{\|\phi\|}{1-\sigma_{P}} .
$$


This means that $\bar{\lambda}(\boldsymbol{\phi}, \boldsymbol{P}[\boldsymbol{s}])$ is a bounded function of $s$.

With $s_{1} \in \mathcal{B}^{n}$ and $s_{2} \in \mathcal{B}^{n}$, let us define $\boldsymbol{\lambda}_{1}=\overline{\boldsymbol{\lambda}}\left(\boldsymbol{\phi}, \boldsymbol{P}\left[s_{1}\right]\right)$ and $\boldsymbol{\lambda}_{2}=\overline{\boldsymbol{\lambda}}\left(\phi, P\left[s_{2}\right]\right)$. From the definition (56), the following relations are satisfied:

$$
\lambda_{1}-\boldsymbol{P}\left[s_{1}\right]\left|\boldsymbol{\lambda}_{1}\right|=\phi, \quad \boldsymbol{\lambda}_{2}-\boldsymbol{P}\left[s_{2}\right]\left|\boldsymbol{\lambda}_{2}\right|=\phi .
$$

Eliminating $\phi$ from the above results in the following:

$$
\boldsymbol{\lambda}_{1}-\boldsymbol{\lambda}_{2}-\boldsymbol{P}\left[\boldsymbol{s}_{1}\right]\left(\left|\boldsymbol{\lambda}_{1}\right|-\left|\boldsymbol{\lambda}_{2}\right|\right)=\boldsymbol{P}\left[\left|\boldsymbol{\lambda}_{2}\right|\right]\left(\boldsymbol{s}_{1}-\boldsymbol{s}_{2}\right) .
$$

Considering that $\left\|\left|\boldsymbol{\lambda}_{1}\right|-\left|\boldsymbol{\lambda}_{2}\right|\right\| \leq\left\|\boldsymbol{\lambda}_{1}-\boldsymbol{\lambda}_{2}\right\|$ and $\sigma_{P}<1$, one obtains the following:

$$
\begin{aligned}
\left(1-\sigma_{P}\right)\left\|\boldsymbol{\lambda}_{1}-\boldsymbol{\lambda}_{2}\right\| & \leq \| \text { L.H.S. of }(83) \| \\
=\| \text { R.H.S. of }(83) \| & \leq \sigma_{P}\left\|\boldsymbol{\lambda}_{2}\right\|_{\infty}\left\|s_{1}-s_{2}\right\| .
\end{aligned}
$$

Considering (81) and (84), one has the following:

$$
\begin{aligned}
\left\|\boldsymbol{\lambda}_{1}-\boldsymbol{\lambda}_{2}\right\| & \leq \frac{\sigma_{P}\left\|\boldsymbol{\lambda}_{2}\right\|_{\infty}\left\|\boldsymbol{s}_{1}-\boldsymbol{s}_{2}\right\|}{1-\sigma_{P}} \\
& \leq \frac{\sigma_{P}\|\boldsymbol{\phi}\|}{\left(1-\sigma_{P}\right)^{2}}\left\|\boldsymbol{s}_{1}-\boldsymbol{s}_{2}\right\| .
\end{aligned}
$$

Therefore, for any $\varepsilon>0$, choosing $\delta$ so that

$$
0<\delta<\frac{\left(1-\sigma_{P}\right)^{2}}{\sigma_{P}\|\phi\|} \varepsilon
$$

results in that $\left\|s_{1}-s_{2}\right\|<\delta$ implies $\left\|\boldsymbol{\lambda}_{1}-\boldsymbol{\lambda}_{2}\right\|<\varepsilon$. This means that $\bar{\lambda}(\phi, P[s])$ is a continuous function of $s$.

\section{REFERENCES}

[1] P. M. Wensing, A. Wang, S. Seok, D. Otten, J. Lang, and S. Kim, "Proprioceptive actuator design in the MIT Cheetah: Impact mitigation and high-bandwidth physical interaction for dynamic legged robots," IEEE Transactions on Robotics, vol. 33, no. 3, pp. 509-522, 2017.

[2] Y. Sim and J. Ramos, "The dynamic effect of mechanical losses of transmissions on the equation of motion of legged robots," RAL, 2021.

[3] M. T. S. Aung and R. Kikuuwe, "Stability enhancement of admittance control with acceleration feedback and friction compensation," Mechatronics, vol. 45, pp. 110-119, 2017.

[4] M. T. S. Aung, R. Kikuuwe, and M. Yamamoto, "Friction compensation of geared actuators with high presliding stiffness," Transactions of ASME: Journal of Dynamic Systems, Measurement, and Control, vol. 137, no. 1, p. 011007, 2015.

[5] M. Iwatani and R. Kikuuwe, "Some improvements in elastoplastic friction compensator," SICE Journal of Control, Measurement, and System Integration, vol. 10, no. 3, pp. 141-148, 2017.

[6] A. Wang and S. Kim, "Directional efficiency in geared transmissions: Characterization of backdrivability towards improved proprioceptive control," in Proceedings of the 2015 IEEE International Conference on Robotics and Automation, 2015, pp. 1055-1062.

[7] A. D. Wang, "Directional impedance of geared transmissions," Master's thesis, Massachusetts Institute of Technology, 2012.

[8] M. Plooji, G. Mathijssen, P. Cherelle, D. Lefeber, and B. Vanderborght, "Lock your robot: A review of locking devices in robotics," Robotics Automation Magazine, vol. 22, no. 1, pp. 106-117, 2005.

[9] M. E. Dohring, E. Lee, and W. S. Newman, "A load-dependent transmission friction model: Theory and experiments," in Proceedings of the 1993 IEEE International Conference on Robotics and Automation, 1993, pp. 430-436.

[10] A. Mablekos-Alexiou, L. da Cruz, and C. Bergeles, "Friction-inclusive modeling of sliding contact transmission systems in robotics," IEEE Transactions on Robotics, vol. 37, no. 4, pp. 1252-1267, 2021.

[11] T.-J. Yeh and F.-K. Wu, "Modeling and robust control of worm-gear driven systems," Simulation Modelling Practice and Theory, vol. 17, pp. 767-777, 2009.

[12] D. C. May, S. Jayasuriya, and B. W. Mooring, "Modeling and control of a manipulator joint driven through a worm gear transmission," Journal of Vibration and Control, vol. 6, pp. 85-111, 2000.
[13] A. Diez-Ibarbia, A. Fernandez-Del-Rincon, P. Garcia, A. De-Juan, M. Iglesias, and F. Viadero, "Assessment of load dependent friction coefficients and their influence on spur gears efficiency," Meccanica, 2018.

[14] H. Matsuki, K. Nagan, and Y. Fujimoto, "Bilateral drive gear - a highly backdriavable reduction gearbox for robotic actuators," IEEE/ASME Transactions on Mechatronics, vol. 24, no. 6, pp. 2661-2673, 2019.

[15] B. Brogliato, Nonsmooth Mechanics: Models, Dynamics and Control, 3rd ed. Springer, 2016

[16] T. Yada, "Review of gear efficiency equation and force treatment," JSME International Journal, Series C, Dynamics, Control, Robotics, Design and Manufacturing, vol. 40, no. 1, pp. 1-8, 1997.

[17] G. V. Smirnov, Introduction to the Theory of Differential Inclusions. Providence, Rhode Islands, USA: American Mathematical Society, 2002.

[18] J.-B. Hiriart-Urruty and C. Lemaréchal, Fundamentals of Convex Analysis. Springer, 2001.

[19] V. Acary and B. Brogliato, Numerical Methods for Nonsmooth Dynamical Systems: Applications in Mechanics and Electronics, ser. Lecture Notes in Applied and Computational Mechanics. Springer, 2008, vol. 35 .

[20] A. Cellina, "On uniqueness almost everywhere for monotonic differential inclusions," Nonlinear Analyais, Theory, Methods \& Applications, vol. 25, no. 9-10, pp. 899-903, 1995.

[21] A. Blumentals, B. Brogliato, and F. Bertails-Descoubes, "The contact problem in Lagrangian systems subject to bilateral and unilateral constraints, with or without sliding Coulomb's friction: a tutorial," Multibody System Dynamics, vol. 38, pp. 43-76, 2016.

[22] V. Acary, O. Bonnefon, and B. Brogliato, "Time-stepping numerical simulation of switched circuits within the nonsmooth dynamical systems approach," IEEE Transactions on Computer-Aided Design of Integrated Circuits and Systems, vol. 29, no. 7, pp. 1042-1055, 2010.

[23] M. Anitescu and F. A. Potra, "Formulating dynamic multi-rigid-body contact problems with friction as solvable linear complementarity problems," Nonlinear Dynamics, vol. 14, no. 3, pp. 231-247, 1997.

[24] D. E. Stewart and J. C. Trinkle, "An implicit time-stepping scheme for rigid body dynamics with inelastic collisions and Coulomb friction," International Journal for Numerical Methods in Engineering, vol. 39, no. 15 , pp. 2673-2691, 1996.

[25] R. Kikuuwe, N. Takesue, A. Sano, H. Mochiyama, and H. Fujimoto, "Admittance and impedance representations of friction based on implicit Euler integration," IEEE Transactions on Robotics, vol. 22, no. 6, pp. 1176-1188, 2006.

[26] F. Facchinei and J.-S. Pang, Finite-Dimensional Variational Inequalities and Complementarity Problems, Volume I. Springer, 2003. 